\title{
Solution of the Multichannel Coqblin-Schrieffer Impurity Model and Application to Multi-Level systems
}

\author{
Andrés Jeredt \\ University of Oxford, Department of Physics, Theoretical Physics, 1 Keble Road, \\ Oxford OX1 3NP, United Kingdom \\ Natan Andrei \\ Department of Physics and Astronomy, Rutgers University, Piscataway, NJ 08855, USA \\ Gergely Zaránd \\ Institute of Physics, Technical University of Budapest, \\ H 1521 Budafoki út 8., Budapest, Hungary
}

\begin{abstract}
2 Residual entropy - the fixed point 11
3 First order - the neighborhood of the fixed point $\ldots \ldots \ldots . \ldots 12$

Coq ptin schrieffer model and a detailed analysis of some physical applications of the model are given. As in the usuah multichan mel Kondu model a variety of Fermil liquid and nom-Fermi liquil (NFL) fir pints is fond, the impurity representation, $\mu$. For $\mu=f$ we find a Fermi liquid For $f>\mu$ the impurity is overscreened and the model has NFL properties. The form the NFL behavior takes depends on the $N$ and $f$ : for $N \leq f$ the specific heat and the susceptibility are dominated by the NFL contributions, for $N>f$ the leading contributions are Fermi-liquid like and the NFL behavior can be seen only to subleading order, while for $N=f$ the behavior is marginal. We also analyze the possibility of physical realizations. We show by a detailed renormalization group and $1 / f$ analysis that the tunneling $N$-state problem can be mapped into the $S U(N) \times S U(f)$ exchange model, and discuss the subtle differences between the two models. As another physical realization we suggest a double quantum dot strufture that can be described by means of an $S U(3) \times S U(2)$ model if the parameters of the dots are tuned appropriately.

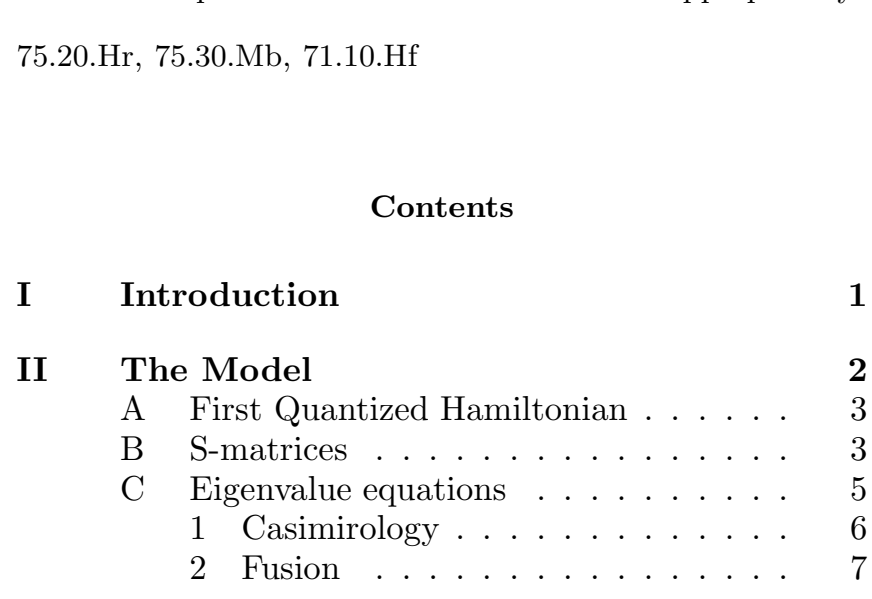

4 Specific heat and finite temperature susceptibility ............. 13

D Channel Anisotropy ............ 15

E Impurity in a higher dimensional representation ............ 15

IV Numerical Analysis $\quad 16$

A Procedure ............... 17

B $\quad$ Results . . . . . . . . . . . . . . 17

1 Entropy . . . . . . . . . . . . . 17

2 specific heat . . . . . . . . . . 18

3 Susceptibility . . . . . . . . . . 18

4 Wilson Ratio. . . . . . . . . . . . . 20

5 Channel Anisotropy . . . . . . . . . 21

V Physical realizations of the $S U(N) \times$ $S U(f)$ model 22

A The N-level system model and its lowenergy fixed point ......... 22

B Stability analysis of the $S U(N) \times S U(f)$ fixed point of the NLS model in the large $f$ limit

C Scaling of the physical quantities of the NLS model in the large $f$ limit .

D Discussion of the possible physical realizations of the NLS model . . . . . . 27

\begin{tabular}{lll}
\hline VI & Conclusions & 28 \\
\hline
\end{tabular}

VII Acknowledgments 29
\end{abstract}

III Thermodynamics

A Thermodynamic Bethe Ansatz equations 8

B Scaling limit .............. 10

C Asymptotic solutions - low temperature properties .............. 10

1 Zeroth order- the fixed point . . . . 10

\section{INTRODUCTION}

The multichannel Kondo model is the simplest impurity model with non-Fermi liquid behavior. Originally introduced to describe "real metals" with magnetic impurities, its applications go beyond the study of dilute magnetic alloys. For instance, it has been known for 
some time that systems consisting of heavy atoms tunneling between two neighboring sites and interacting with conduction electrons are a realization of the two-channel Kondo modell. Another realization is thequadrupolar Kondo effect in context of heavy fermions 3 . A detailed account of various aspects and applications of the multichannel Kondo model is given in Ref. 4 .

For materials such as $P b_{1-x} G e_{x} T e$ or $K_{1-x} L i_{x} C l$ alloys, the tunneling may occur between an arbitrary number of levels. Such systems could be modeled using a multichannel version of the Cofblin-Schrieffer model, a $S U(N) \times S U(f)$ Kondo model 5 . Here, $N$ is the number of spin degrees of freedom, and $f$ is the number of channels, or flavor degrees of freedom.

In this article we present an exact solution of the $S U(N) \times S U(f)$ Kondo model, and study the thermodynamic properties of the system. We obtain the leading exponents for the impurity contribution to the magnetic susceptibility and specific heat for arbitrary $N$ and $f$. We also discuss the effects of channel anisotropy, which might drive the system from a fixed point with $\gamma \equiv f / N>1$ to a new fixed point where $\gamma^{\text {eff }}<1$.

\section{THE MODEL}

The Multichannel Coqblin-Schrieffer (MCCS) model describes electrons carrying two sets of internal degrees of freedom, to be denoted spin and flavor (or channel number), interacting with an impurity carrying only spin. The impurity is localized at a point chosen to be the origin. The Hamiltonian reads,

$$
\begin{aligned}
\mathcal{H} & =-i \sum_{a}^{N} \sum_{m}^{f} \int_{-\infty}^{\infty} \psi_{a, m}^{\dagger}(x) \partial_{x} \psi_{a, m}(x) d x \\
& +2 \sum_{m}^{f} J_{m} \sum_{\alpha}^{N^{2}-1} \sum_{a, b}^{N} \psi_{a, m}^{\dagger}(0)\left(T_{\alpha}^{(\square)}\right)_{a, b} \psi_{b, m}(0) \\
& \times \sum_{a^{\prime}, b^{\prime}}^{\operatorname{dim}(\mu)} \chi_{a^{\prime}}^{\dagger}\left(T_{\alpha}^{(\mu)}\right)_{a^{\prime}, b^{\prime}} \chi_{b^{\prime}}
\end{aligned}
$$

Both $\psi_{a, m}^{\dagger}(x)$ and $\chi_{a}^{\dagger}$ are fermionic fields, the former creates an electron at $x$ with spin index $a$ and flavor index $m$, while the latter creates the impurity at $x=0$. Imposing the condition $\sum_{a} \chi_{a}^{\dagger} \chi_{a}=1$, we have that $\chi_{a^{\prime}}^{\dagger}\left(T_{\alpha}^{(\mu)}\right)_{a^{\prime}, b^{\prime}} \chi_{b^{\prime}}$ represents the impurity spin operator in a representation of $S U(N)$ specified by a particular choice of the matrices $T_{\alpha}^{(r e p)}$, where the index $\alpha$ runs from 1 to $N^{2}-1$, the number of generators of $S U(N)$. We will restrict ourselves to the case in which the electrons are in the fundamental representation (denoted by $(\square)$ ), and the impurity is in the totally symmetric representation obtained from the direct product of $\mu$ fundamental representations.
The physical realizations discussed in the present paper correspond to the simplest case, $\mu=1$; the investigation of the $\mu>1$ cases gives us important insight into the general structure of the model and allows, in particular, for a comparison with the results obtained for the multichannel Kondo model with impurity spin $S>1 / 2$.

In most of this paper we will study the isotropic model, $J_{m}=J$, with the symmetry $U(1)^{\text {charge }} \times S U(N)^{\text {spin }} \times$ $S U(f)^{\text {flavor }}$. We will also assume that the different flavor levels are equally populated, $N^{e}=f N_{0}$.

In what follows shall solve the complete model and among other things, study its low-energy physics. As is well known, the low energy behavior of a system can often be described in terms of effective hamiltonians, that are simpler than the starting hamiltonian; these are usually referred to as fixed points. We shall determine their properties from the exact solution. We shall find that the model possesses a variety of fixed points (or low energy regimes), whose nature depends on the symmetry structure in the flavor sector and on the spin representation $(\mu)$, generalizing the familiar $N=2$ case (the multichannel Kondo mode 1,6611.31. As previously, we shall identify the mechanism underlying the appearance of these fixed points as dynamical fusion by which electrons form spin complexes whose interaction with the impurity leads to a new behavior in the infrared 6 . Each complex consists of $f$ electrons fused into a local objects that transforms according to one-row Young Tableaux of length $f$.

Within the Bethe-Ansatz approach a precise description of the formation of these composites can be given. The linearized hamiltonian propagates separately the charge-spin-flavor degrees of freedom that make up the electron. Therefore the effect of flavor on the spin degrees of freedom is recovered only in the physical space. To follow the dynamic coupling of spin and flavor we add some curvature which maintains the identity of the electron while allowing its components to interact. It has the form $H_{\Lambda}=\frac{1}{2 \Lambda} \sum_{a} \sum_{m} \int_{-\infty}^{\infty} \psi_{a, m}^{\dagger}(x) \partial_{x}^{2} \psi_{a, m}(x) d x$, where $\Lambda$ is the curvature scale which is sent to infinity at the end of the calculation. Adding this term allows for the formation of bound states in the flavor singlet channel, which interact strongly with the impurity, and determine the low-energy dynamics even after the curvature is removed. A close analogy is a small magnetic field introduced to probe for magnetization, which may survive after the field is removed. Imposing a cut-off $D$ on the momentum variables guarantees the finiteness of the energy. Other terms need to be added to the hamiltonian to maintain integrability, terms which we shall see below are irrelevant.

Already for free fields the resulting theory is quite in volved, and even the counting of states is not triviall. Nevertheless, the charge-spin-flavor separated basis is the natural one for the non-interacting problem, as we shall see later: it is the form to which the eigenstates tend when the interaction is turned off. We thus introduce the following elements: 
- A second derivative term with a curvature scale, $\Lambda$,

$$
H_{\Lambda}=\frac{1}{2 \Lambda} \sum_{a} \sum_{m} \int_{-\infty}^{\infty} \psi_{a, m}^{\dagger}(x) \partial_{x}^{2} \psi_{a, m}(x) d x
$$

which breaks charge-spin-flavor (CSF) separation of the linear spectrum. Once the electron composites are formed, and the low-energy spectrum of the theory is identified, the scale is taken to infinity.

Adding the term (2.2) also imposes restrictions on the form of the eigenstates which can be expressed in terms of the following counterterms without which the model is not integrable for finite $\Lambda$ :

- An electron-electron interaction term, of the form

$2 \tilde{J} \sum_{m, m^{\prime}} \sum_{a, a^{\prime}} \int_{-\infty}^{\infty} \psi_{a, m}^{\dagger}(x) \psi_{a^{\prime}, m^{\prime}}^{\dagger}(x) \psi_{a, m^{\prime}}(x) \psi_{a^{\prime}, m}(x) d x$

When no impurity is present $\tilde{J}$ can be chosen arbitrarily since the term has no effect on the linear spectrum. The linearized spectrum has a large degeneracy, and the inclusion of (2.2) and (2.3) will provide a way to find the eigenstates.

- A counterterm $H_{c c}$, of the form

$$
H_{c c}=-\frac{1}{\Lambda} \sum_{m}^{f} \sum_{a}^{N} \int_{-\infty}^{\infty} \psi_{a, m}^{\dagger}(x) V(x) \psi_{a, m}(x) d x
$$

with

$$
V(x)=\frac{x}{|x|}\left(\delta^{\prime}\left(x^{+0}\right)+\delta^{\prime}\left(x^{-0}\right)\right),
$$

needs to be added to the Hamiltonian in order to preserve integrability at the origin; this term vanishes once the curvature is removed, and plays no further role in the problem.

\section{A. First Quantized Hamiltonian}

A general Fock state of $N^{e}$ electrons and one impurity can be written in the following form:

$$
\begin{aligned}
\mid F> & =\sum_{\left\{m_{j}\right\}} \sum_{\left\{a_{j}\right\}, b} \int_{-\infty}^{\infty}\left(\prod_{j} d x_{j}\right) F_{\left\{a_{j}\right\}, b}^{\left\{m_{j}\right\}}\left(\left\{x_{j}\right\}\right) \chi_{b}^{\dagger}(0) \\
& \times \prod_{j=1}^{N_{e}} \psi_{a_{j}, m_{j}}^{\dagger}\left(x_{j}\right) \mid 0>.
\end{aligned}
$$

In order for it to be an eigenstate the amplitudes $F$ must satisfy the equation $h F=E F$, where the differential operator $h$, known as the first quantized form of the Hamiltonian, takes the form

$$
\begin{aligned}
h & =\sum_{j=1}^{N^{e}}\left\{-i \partial_{j}+\frac{1}{2 \Lambda} \partial_{j}^{2}+2 J \delta\left(x_{j}\right) \sum_{\alpha}^{N^{2}-1}\left(T_{\alpha, j}^{(\square)}\right)\left(T_{\alpha}^{(\mu)}\right)\right\} \\
& +\sum_{l<j} 2 \tilde{J} \delta\left(x_{l}-x_{j}\right)\left(P_{l j}-\mathcal{P}_{j l}\right)-\sum_{j=1}^{N^{e}} \frac{1}{\Lambda} V\left(x_{j}\right),
\end{aligned}
$$

with $P_{j l}\left(\mathcal{P}_{j l}\right)$ the spin(flavor) exchange operator,

$$
\begin{aligned}
P_{a b, c d} & =\delta_{a d} \delta_{b c}, \\
\mathcal{P}_{m_{1} m_{2}, m_{3} m_{4}} & =\delta_{m_{1} m_{4}} \delta_{m_{2} m_{3}} .
\end{aligned}
$$

The fundamental representation $(\square)$ is carried by the electron $j$ and the $(\mu)$ representation by the impurity. When the latter is also in the fundamental representation i.e. $\mu=1$ the hamiltonian can be rewritten as,

$$
\begin{aligned}
h & =\sum_{j=1}^{N^{e}}-i \partial_{j}+\left(\Lambda^{-1}\right) \partial_{j}^{2}+2 J \delta\left(x_{j}\right) P_{j 0} \\
& +\sum_{l<j} 2 \tilde{J} \delta\left(x_{l}-x_{j}\right)\left(P_{l j}-\mathcal{P}_{j l}\right)+\sum_{j=1}^{N^{e}} \frac{1}{\Lambda} V\left(x_{j}\right) .
\end{aligned}
$$

\section{B. S-matrices}

We will assume for now that both the electrons and the impurity are in the fundamental representation of $S U(N)$. The eigenstate amplitudes are combinations of plane waves with pseudo-momenta $k_{j},\left(j=1, \ldots, N^{e}\right)$, and have coefficients that depend on the ordering of the electrons, and on the spin and the flavor indices. These coefficients are related through products of electronimpurity and electron-electron S-matrices that we will derive now. Consider first the wavefunction describing one electron (denote it by $j$ ) interacting with the impurity (denote it by 0 ),

$F_{a_{j}, a_{0}}^{m_{j}}\left(x_{j}\right)=e^{i k_{j} x_{j}}\left(A_{a_{j}, a_{0}}^{m_{j}} \theta\left(-x_{j}\right)+B_{a_{j}, a_{0}}^{m_{j}} \theta\left(x_{j}\right)\right)$.

Applying $h$ to it we have (we drop the indices in the amplitudes)

$$
\begin{aligned}
h F\left(x_{j}\right)= & \left(k_{j}-\frac{k_{j}^{2}}{2 \Lambda}\right) F\left(x_{j}\right) \\
& \left(-i\left(1-\frac{k_{j}}{\Lambda}\right)(B-A)+J P_{j 0}(B+A)\right) \delta\left(x_{j}\right) \\
& -\frac{1}{2 \Lambda}(B-A) \delta^{\prime}\left(x_{j}\right) e^{i k_{j} x_{j}}+\frac{1}{\Lambda} V\left(x_{j}\right) F\left(x_{j}\right) .
\end{aligned}
$$

$F$ is an eigenstate of $h$, with eigenvalue $E_{j}=k_{j}\left(1-\frac{k_{j}}{2 \Lambda}\right)$, if the terms in the second and third lines in (2.7) vanish. The last two terms cancel each other due to the form of (2.5). The terms in the second line of (2.7) cancel if the amplitudes $A$ and $B$ are related by the electron-impurity S-matrix $B=S_{j 0} A$, where $S_{j 0}=\left(S_{j 0}\right)_{a_{j}, a_{0}}^{a_{j}^{\prime}, a_{0}^{\prime}}$ is given by 


$$
\begin{aligned}
S_{j 0} & =\frac{i\left(1-k_{j} / \Lambda\right)+J P_{j 0}}{i\left(1-k_{j} / \Lambda\right)-J P_{j 0}} \\
& =\left(\frac{i\left(1-k_{j} / \Lambda\right)+J}{i\left(1-k_{j} / \Lambda\right)-J}\right)\left(\frac{i\left(1-k_{j} / \Lambda\right)+J P_{j 0}}{i\left(1-k_{j} / \Lambda\right)+J}\right)^{2} .
\end{aligned}
$$

Defining

$$
c \equiv \frac{2 J}{1-J^{2}}, \quad g(x) \equiv \frac{1-x}{1-J^{2}}\left(1-\frac{J^{2}}{(1-x)^{2}}\right),
$$

we can write

$$
S_{j 0}=e^{-i \arctan \frac{c}{g\left(k_{j} / \Lambda\right)}}\left(\frac{g\left(k_{j} / \Lambda\right)-i c P_{j 0}}{g\left(k_{j} / \Lambda\right)-i c}\right),
$$

(notice that $\arctan c=2 \arctan J$ ). Eventually we will send the cutoff to infinity. Therefore, expanding $g(k / \Lambda)$ to first order in $1 / \Lambda$

$$
g(k / \Lambda) \sim 1-\left(\frac{1+J^{2}}{1-J^{2}}\right) \frac{k}{\Lambda},
$$

we have

$$
S_{j 0} \sim e^{i \arctan \frac{c}{1+\lambda_{j}}}\left(\frac{\lambda_{j}-1+i c P_{j 0}}{\lambda_{j}-1+i c}\right),
$$

where

$$
\lambda_{j}=\left(\frac{1+J^{2}}{1-J^{2}}\right) \frac{k_{j}}{\Lambda},
$$

In the scaling limit, $J$ and $c$ have the same scaling behavior.

We now consider the case of two electrons. We generalize the procedure followed in the case on one electron: divide the configuration space into regions inside each of which there is no interaction and the wavefunction is a superpositions of plane waves. They are six such regions in this case, corresponding to the ordering of three objects: two electrons and an impurity, and we label them by permutations $Q \in S_{3}$. For example, the element $Q=(1,0,2)$ labels the region where electron 1 is to the left of the impurity and electron 2 is to its right. We also introduce the notation $\theta\left(x_{Q}\right)$ to denote a function that takes the value 1 in the region $Q$ and zero elsewhere.

The two electron wave function is then of the form (Bethe-Ansatz),

$$
F_{a}^{m}(x)=\mathcal{A} e^{i\left(k_{1} x_{1}+k_{2} x_{2}\right)} \sum_{Q} \theta\left(x_{Q}\right) A_{a, m}^{Q},
$$

where $m=\left(m_{1}, m_{2}\right)$ and $a=\left(a_{1}, a_{2}, a_{0}\right)$ and $\mathcal{A}$ is the antisymmetrizer. The amplitudes in the various regions are connected by $S$-matrices, e.g. $S^{01} A^{012}=A^{102}$, where $S^{01}$, the electron- impurity $S$-matrix has been already determined in the one electron problem. For this Ansatz to be consistent it must satisfy the Yang-Baxter Relations,

$$
S^{i j} S^{i 0} S^{j 0}=S^{j 0} S^{i 0} S^{i j} .
$$

guaranteeing that the two paths from $(1,2,0)$ to $(0,2,1)$ yield the same answer.

What is the electron-electron $S$-matrix, $S^{i j}$ ? There is no direct electron-electron interaction term in the Hamiltonian (2.1), and one may be tempted to adopt the naive choice $S^{\imath \jmath}=I$ for the scattering matrix of electrons $i$ and $j$. Nevertheless, electron correlations are induced through the impurity. These show up immediately, since the naive choice does not satisfy the Yang-Baxter Relations: $S^{j 0}$ and $S^{i 0}$ do not commute. This non commutativity captures some important aspects of the model: after electron $i$ crossed the impurity the latter is left in a different state than before. Hence the state in which electron $j$ finds the impurity depends on whether it crosses the impurity before or after electron $i$. Herein lies the difference between a system of electrons interacting with a fixed potential (a one-body problem since all electron see the same potential) and a Kondo system, where the impurity correlates the motion of all electrons.

Are we allowed to introduce a scattering matrix $S^{i j}$ to satisfy the Yang-Baxter Relations? We proceed now to show that this is indeed the case, namely, the introduction of an electron-electron scattering matrix would not modify the original problem we set out to solve. Consider first the space of free electrons with a linearized Hamiltonian. The space is highly degenerate: for example the energy $E=k_{1}+k_{2}$ in the two electron space corresponds to a wave function $F=$ $\sum_{q} e^{i\left(k_{1}+q\right) x_{1}+i\left(k_{2}-q\right) x_{2}} A_{q}$ for any choice of coefficients $A_{q}$. Equivalently, we can pick a basis of the form $F=e^{i k_{1} x_{1}+i k_{2} x_{2}}\left(\theta\left(x_{1}-x_{2}\right)+S^{12} \theta\left(x_{2}-x_{1}\right)\right) A$. The choice of $S^{12}$ is arbitrary in the two electron space, but if we wish to proceed to construct three (and more) electron wavefunctions then the scattering matrices satisfy must satisfy the YBE for electrons, $S^{i j} S^{i k} S^{j k}=S^{j k} S^{i k} S^{i j}$. When the Kondo interaction is turned on, the matrix $S^{j 0}$ is fixed by the interaction, which in turn picks the electron basis through the Yang-Baxter Relations (2.10).

When the cut-off is present part of the degeneracy is removed already at the free electron level but the procedure still goes through. Consider the model for two electrons away from the impurity.

$$
\begin{aligned}
h & =-i \partial_{j}-i \partial_{l}+\frac{1}{2 \Lambda} \partial_{j}^{2}+\frac{1}{2 \Lambda} \partial_{l}^{2} \\
& +2 \tilde{J} \delta\left(x_{l}-x_{j}\right)\left(P_{l j}-\mathcal{P}_{l j}\right) .
\end{aligned}
$$

This cut-off Hamiltonian is in the same universality class as the free linearized hamiltonian and possesses the same spectrum when the cut-off is sent to infinity; its particular form was chosen so that the S-matrix it defines does indeed satisfy the (2.10). Again, we divide configuration space into two regions:

$$
\begin{aligned}
& F_{\left\{a_{j}, a_{l}\right\}}^{\left\{m_{j}, m_{l}\right\}}\left(x_{j}, x_{l}\right)= \\
& e^{i\left(k_{j} x_{j}+k_{l} x_{l}\right)}\left(A_{\left\{a_{j}, a_{l}\right\}}^{\left\{m_{j}, m_{l}\right\}} \theta\left(x_{l}-x_{j}\right)+B_{\left\{a_{j}, a_{l}\right\}}^{\left\{m_{j}, m_{l}\right\}} \theta\left(x_{j}-x_{l}\right)\right),
\end{aligned}
$$


and study the eigenvalue equation $h F=E F$. We have

$$
\begin{aligned}
h F= & \left(\left(k_{j}-\frac{k_{j}^{2}}{2 \Lambda}\right)+\left(k_{l}-\frac{k_{l}^{2}}{2 \Lambda}\right)\right) F \\
& +(-i(B-A)-i(A-B) \\
& \left.+i\left(\frac{k_{j}}{\Lambda}-\frac{k_{l}}{\Lambda}\right)(B-A)\right) \delta\left(x_{j}-x_{l}\right) e^{i\left(k_{j}+k_{l}\right) x_{j}} \\
& +\tilde{J}\left(P_{j l}-\mathcal{P}_{j l}\right)(A+B) \delta\left(x_{j}-x_{l}\right) e^{i\left(k_{j}+k_{l}\right) x_{j}} \\
& +\frac{1}{2 \Lambda}((B-A)+(A-B)) \delta^{\prime}\left(x_{j}-x_{l}\right) e^{i\left(k_{j} x_{j}+k_{l} x_{l}\right)} .
\end{aligned}
$$

The last line is identically zero; counterterms of the form (2.4) are only necessary when the particles involved have different velocities. The rest of the terms proportional to $\delta\left(x_{j}-x_{l}\right)$ cancel if the amplitudes in the different regions are related by the following electron-electron S-matrix,

$$
S_{j l}=\frac{i \alpha_{j l}+\tilde{J}\left(P_{j l}-\mathcal{P}_{j l}\right)}{i \alpha_{j l}-\tilde{J}\left(P_{j l}-\mathcal{P}_{j l}\right)},
$$

where $\alpha_{j l} \equiv\left(k_{l}-k_{j}\right) / \Lambda$. Such a S-matrix can be written as

$$
S_{j l}=\frac{\alpha_{j l}-2 i \tilde{J} P_{j l}}{\alpha_{j l}-2 i \tilde{J}} \frac{\alpha_{j l}+2 i \tilde{J} \mathcal{P}_{j l}}{\alpha_{j l}+2 i \tilde{J}}
$$

Choosing

$$
\tilde{J}=\frac{J}{1+J^{2}},
$$

allows us to express the $S$-matrix as,

$$
S_{j l}=\frac{\lambda_{j}-\lambda_{l}+i c P_{j l}}{\lambda_{j}-\lambda_{l}+i c} \frac{\lambda_{j}-\lambda_{l}-i c \mathcal{P}_{j l}}{\lambda_{j}-\lambda_{l}-i c} .
$$

The S-matrices (2.9,2.14) satisfy the Yang-Baxter conditions (2.10), and also

$$
S^{i j} S^{i k} S^{j k}=S^{j k} S^{i k} S^{i j}
$$

assuring that we were able to generate a cut-off version of the Hamiltonian while maintaining integrability.

The cut-off scheme we introduced generates a flavor component in the electron-electron $S$-matrix. Clearly it captures the interaction among electrons induced by the impurity. More so, already for the free hamiltonian $\mathcal{H}_{0}=-i \sum_{a}^{N} \sum_{m}^{f} \int_{-\infty}^{\infty} d x \psi_{a, m}^{\dagger}(x) \partial_{x} \psi_{a, m}(x)$ a non trivial S-matrix must be introduced if we choose an $S U(N) \times S U(f)$ invariant basis (which is appropriate for a subsequent inclusion of an impurity interaction) rather than the simpler $\operatorname{SL}(f N)$. A careful counting of states can be carried out to show that all expected states then appear with the correct degeneracies. It is instructive that this would not be the case for the naive choice $S_{(\text {flavor })}^{i j}=I$.

The energy eigenvalues of a $N^{e}$-electron state are a generalization of the first line of (2.12). They are of the form

$$
E=\sum_{j=1}^{N^{e}} k_{j}\left(1-\frac{k_{j}}{2 \Lambda}\right)
$$

\section{Eigenvalue equations}

In order to determine the spectrum, we impose periodic boundary conditions, and solve the correspond ing eigenvalue problem. The procedure is standard 10 and we skip here the details. The result is contained in the Bethe Ansatz Equations (BAE) which we proceed to write down. Each of the degrees of freedom - charge, spin and flavor - is described by a set of variables whose number depends on the symmetry of the particular state. The charge degrees of freedom are given by the set $\left\{k_{j}, j=\right.$ $\left.1, \ldots, N^{e}\right\}$. The spin degrees of freedom are parameterized by the sets $\left\{\chi_{\gamma}^{r}, \gamma=1, \ldots, M^{r} ; r=1, \ldots, N ; M^{N}=0\right\}$. Finally, the flavor degrees of freedom are represented by the sets $\left\{\omega_{\gamma}^{r}, \gamma=1, \ldots, \bar{M}^{r} ; r=1, \ldots, f ; \bar{M}^{f}=0\right\}$. The set of integers $M^{r} ; r=1, \ldots, N-1$ specify the symmetry of the spin component of the wave function given by a $S U(N)$ Young tableau with the length $l^{r}$ of the $r$ th row given by $l^{r}=M^{r}-M^{r+1}, M^{N}=0, M^{0}=N^{e}+1$. Similarly, the quantum numbers $\left\{\bar{M}^{r}\right\}$ specify the symmetry of the flavor component.

The equations are

$$
\begin{aligned}
e^{i k_{j} L} & =\prod_{\gamma=1}^{M^{1}} \frac{\chi_{\gamma}^{1}-\left(1-\lambda_{j}\right)+i \frac{c}{2}}{\chi_{\gamma}^{1}-\left(1-\lambda_{j}\right)-i \frac{c}{2}} \prod_{\gamma=1}^{\bar{M}^{1}} \frac{\omega_{\gamma}^{1}-\lambda_{j}+i \frac{c}{2}}{\omega_{\gamma}^{1}-\lambda_{j}-i \frac{c}{2}} \\
- & \prod_{\beta=1}^{\bar{M}^{r}} \frac{\omega_{\gamma}^{r}-\omega_{\beta}^{r}+i c}{\omega_{\gamma}^{r}-\omega_{\beta}^{r}-i c}=\prod_{t=r \pm 1} \prod_{\beta=1}^{M^{t}} \frac{\omega_{\gamma}^{r}-\omega_{\beta}^{t}+i \frac{c}{2}}{\omega_{\gamma}^{r}-\omega_{\beta}^{t}-i \frac{c}{2}} ; \\
& +\prod_{\beta=1} \frac{\omega_{\gamma}^{1}-\omega_{\beta}^{1}+i c}{\omega_{\gamma}^{1}-\omega_{\beta}^{1}-i c}=\prod_{j=1}^{N^{e}} \frac{\omega_{\gamma}^{1}-\lambda_{j}+i \frac{c}{2}}{\omega_{\gamma}^{1}-\lambda_{j}-i \frac{c}{2}} \\
\times & \prod_{\beta=1}^{\bar{M}^{2}} \frac{\omega_{\gamma}^{1}-\omega_{\beta}^{2}+i \frac{c}{2}}{\omega_{\gamma}^{1}-\omega_{\beta}^{2}-i \frac{c}{2}}, \\
- & \prod_{\beta=1}^{M^{r}} \frac{\chi_{\gamma}^{r}-\chi_{\beta}^{r}+i c}{\chi_{\gamma}^{r}-\chi_{\beta}^{r}-i c}=\prod_{t=r \pm 1} \prod_{\beta=1}^{M^{t}} \frac{\chi_{\gamma}^{r}-\chi_{\beta}^{t}+i \frac{c}{2}}{\chi_{\gamma}^{r}-\chi_{\beta}^{t}-i \frac{c}{2}} ; \\
& r=2, \ldots, N-1, \\
& =\prod_{\beta=1}^{M^{1}} \frac{\chi_{\gamma}^{1}-\chi_{\beta}^{1}+i c}{\chi_{\gamma}^{1}-\chi_{\beta}^{1}-i c}=\frac{\chi_{\gamma}^{1}+i \frac{c}{2}}{\chi_{\gamma}^{1}-i \frac{c}{2}} \prod_{j=1}^{N^{e}} \frac{\chi_{\gamma}^{1}-\left(1-\lambda_{j}\right)+i \frac{c}{2}}{\chi_{\gamma}^{1}-\left(1-\lambda_{j}\right)-i \frac{c}{2}} \\
& \prod_{\beta=1}^{2} \frac{\chi_{\gamma}^{1}-\chi_{\beta}^{2}+i \frac{c}{2}}{\chi_{\gamma}^{1}-\chi_{\beta}^{2}-i \frac{c}{2}} .
\end{aligned}
$$

The next step is to solve the equations for all possible states, identify the ground state and the low energy excitations. Subsequently, by summing over all excitation energies we shall obtain the partition function.

The BAE contain the cutoff $\Lambda$ which eventually is sent to infinity. We shall find that in this limit the equations reduce to a smaller set once the correct ground state has been identified. It is composed of string-solutions (see below) corresponding to electron composites which interact 
most efficiently with the impurity. To sharpen our intuition we begin by some strong coupling considerations.

\section{Casimirology}

As mentioned, the mechanism underlying the physics of the multichannel Kondo model is the dynamic formation of electron composites. We expect that those configurations are favored which allow minimization of the local interaction at the impurity site. Consider then the general problem of finding the ground state of the following hamiltonian:

$$
J \sum_{a}^{N^{2}-1} T_{a}^{(e)} T_{a}^{(i)}
$$

where the set $\left\{T_{a}^{e}, a=1, \ldots, N^{2}-1\right\}$ is an arbitrary representation of $S U(N)$, and $\left\{T_{a}^{i}, a=1, \ldots, N^{2}-1\right\}$ is the particular representation of the impurity, in our case it will typically be $(\mu)$. In this article we will consider impurities with spin in a totally symmetric representation (for more general representations see12). Each set is normalized: $\operatorname{Tr}\left(T_{a} T_{b}\right)=\frac{1}{2} \delta_{a, b}$.

The largest number of electrons allowed at the origin by the exclusion principle is $N \times f$. This is obtained by placing $N$ electrons in each of the channels. However, such a state is a singlet, both in spin and in flavor, and gives a zero contribution to (2.16). Therefore, the number of electrons that form the composite, $M$, is such that $M \leq(N-1) \times f$. We will show here that if the impurity is in a totally symmetric representation, the electron composite that minimizes (2.16) is made out of $M=(N-1) \times f$ electrons.

We will characterize the different representations of $S U(N)$ by their Young tableaux. The fundamental representation is denoted by a box, $\square$, and the singlet by a point $\bullet$. The totally (anti)symmetric representation resulting from the direct product of $\mu$ representations is denoted by a single (column)row made out of $\mu$ boxes, where in the antisymmetric case we assume $\mu \leq N$.
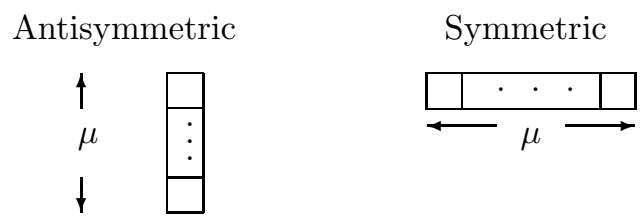

An arbitrary representation resulting from a product of $M$ fundamentals is associated with a Young tableau made up of $M$ boxes, distributed in $k \leq N$ rows. Let $m_{j}$ be the number of boxes in the $j$-th row. Then, $m_{j} \geq m_{j+1}$, $\sum_{j=1}^{k} m_{j}=M$. The corresponding Young tableau will be of the form

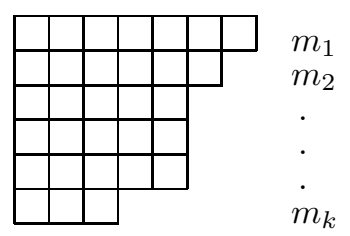

When $N=2$, the interaction $(2.16)$ can be written in terms of conserved quantities

$$
\begin{aligned}
& J \vec{S}^{M e} \cdot \vec{S}^{i}= \\
& \frac{J}{2}\left(S^{t o t}\left(S^{t o t}+1\right)-S^{M e}\left(S^{M e}+1\right)-S^{i}\left(S^{i}+1\right)\right) .
\end{aligned}
$$

The operator $S(S+1)$ is a particular case of the Casimir operator, $C(\Gamma)$, of $S U(N)$, which commutes with all the generators of the group. For arbitrary $N$,

$$
J \sum_{a} T_{a}^{M} T_{a}^{i}=\frac{J}{2}\left(C\left(\Gamma^{T}\right)-C\left(\Gamma^{M}\right)-C\left(\Gamma^{i}\right)\right) .
$$

Given a representation $\Gamma$ of $S U(N)$ with $M$ boxes distributed according to the set $\left\{m_{j}\right\}$, we have 13

$$
C(\Gamma)=\frac{M\left(N^{2}-M\right)}{2 N}+\frac{1}{2} \sum_{j=1}^{k} m_{j}\left(m_{j}+1-2 j\right) .
$$

We can use Young tableaux as an easy way of decomposing the direct product of representations into a direct sum. The procedure is standard (see for instance 14 ).

The electrons will be evenly distributed among the channels, forming a flavor singlet. Hence, the spin of the electron composites is described by a rectangular tableau with $f$ columns and $k \leq N-1$ rows. Multiplying the electron tableau by the impurity tableau we have (in the graphic representation we drop from the tableaux the singlet part consisting of columns of length $N$ ),

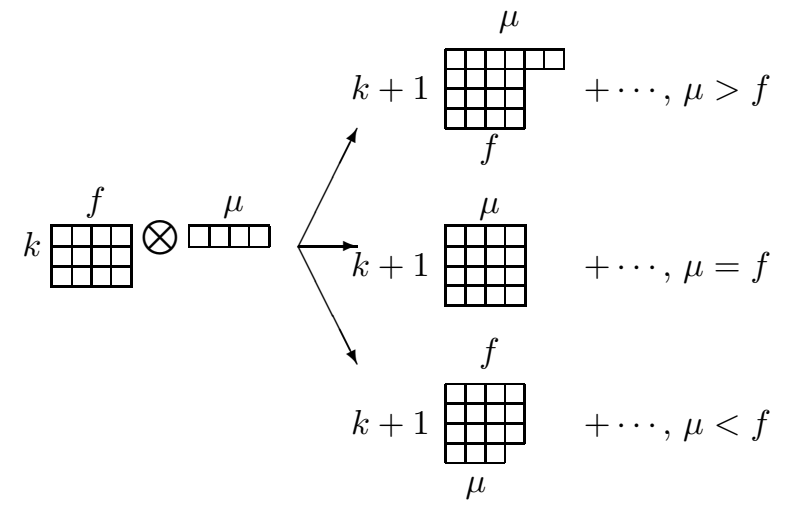

for $k<N-1$. If $k=N-1$, we have 


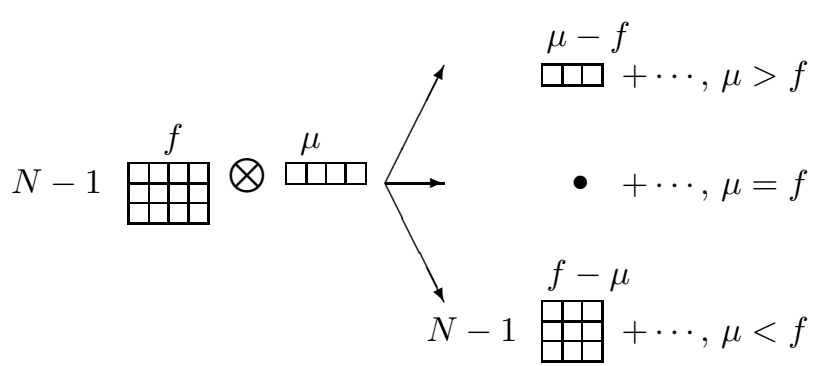

Notice that we have drawn only the terms in the decompositions that give the lowest energy. The energy for all such configurations is given by

$$
J \sum_{a} T_{a}^{M} T_{a}^{i}=-k \frac{\min (\mu, f)}{2 N}(N+\max (\mu, f)) .
$$

Therefore, the energy is minimized when composites of $(N-1) \times f$ electrons are formed.

There are three different situations depending on the value of $\mu / f$, as in the multichannel Kondo problemt. When $\mu>f$, there is underscreening: the electrons cannot screen the impurity completely and the spin configuration is characterized by a Young tableau with one row and $\mu-f$ columns. As in the $N=2$ case, we will see later that such object behaves as a free spin in the Kondo problem. The second case, $\mu=f$, corresponds to complete screening: the electrons and the impurity form a singlet. This is a stable fixed point of the full Hamiltonian with Fermi liquid behavior. Finally, $\mu>f$ corresponds to overscreening: there are more electrons than necessary to screen the impurity. The resulting object corresponds to a tableau with $N-1$ columns and $f-\mu$ rows. This configuration is unstable to the kinetic term, and the fixed point in this case is characterized by Non-Fermi liquid behavior, as we will see later.

\section{Fusion}

We turn now to the dynamics of the full model captured by the BAE. We shall argue that the ground state and low lying excitations lie in a sector of the theory given by solutions of a particular form - $f-$ strings. Solutions of this type are $S U(f)$ flavor singlets - allowing them to have maximally large $S U(N)$ spin. We shall find that this class of excitations is characterized by a scale $T_{0}=D e^{-\frac{2 \pi}{N c}}$. When strings are broken to form flavored excitations we expect them to be characterized by other scales which will tend to infinity as the cut-off is reploved and thus not contribute to the impurity dynamicst.

The formation of composites in flavor corresponds to solutions of the BAE where the charge parameters, $\left\{\lambda_{j}\right\}$, are complex numbers centered around $\left\{\omega_{\gamma}^{1}\right\}$, according to the string hypothesis 8 . Likewise, rank $r$ flavor parameters are themselves centered around rank $r+1$ solutions 6 . The form of the charge parameters is,

$$
\lambda_{\delta}^{q}=\frac{p_{\delta}}{\Lambda}+i c\left(\frac{f+1}{2}-q\right), \quad q=1,2, \ldots, f, \quad p_{\delta} \text { real } .
$$

while the flavor parameters,

$$
\begin{array}{r}
\left\{\omega_{\gamma}^{r}, \gamma=1,2, \ldots, M^{r}\right\}=\left\{p_{A} / \Lambda+i J[(f-r+1) / 2-q] ;\right. \\
\\
q=1,2, \ldots, f-r, A=1, \ldots, N\}
\end{array}
$$

where $r=0,1, \ldots, f-1$. These configurations satisfy the BAE in a trivial manner and induce fusion in the BAE equations as well as in the form of the wavefunctions. A string built on momentum $p$ as its real part induces in the wave function a composite of the form $\exp \left\{-\frac{1}{2} \Lambda J \sum_{j, l}\left|x_{j}-x_{l}\right|+i p\left(x_{1}+\ldots+x_{f}\right)\right\} \times[\ldots]$, which becomes local as $\Lambda \rightarrow \infty$.

Inserting the string configurations into the full BAE we obtain the effective equations governing the impurity spin dynamics. After removing the cutoff they become,

$$
\begin{aligned}
& e^{i f p_{\delta} L}=\prod_{\gamma=1}^{M^{1}} \frac{\chi_{\gamma}^{1}-1+i f \frac{c}{2}}{\chi_{\gamma}^{1}-1-i f \frac{c}{2}}, \\
&-\prod_{\beta=1}^{M^{r}} \frac{\chi_{\gamma}^{r}-\chi_{\beta}^{r}+i c}{\chi_{\gamma}^{r}-\chi_{\beta}^{r}-i c}=\prod_{t=r \pm 1} \prod_{\beta=1}^{M^{t}} \frac{\chi_{\gamma}^{r}-\chi_{\beta}^{t}+i \frac{c}{2}}{\chi_{\gamma}^{r}-\chi_{\beta}^{t}-i \frac{c}{2}} ; \\
&-\prod_{\beta=1}^{M^{1}} \frac{\chi_{\gamma}^{1}-\chi_{\beta}^{1}+i c}{\chi_{\gamma}^{1}-\chi_{\beta}^{1}-i c}=\frac{\chi_{\gamma}^{1}+i \frac{c}{2}}{\chi_{\gamma}^{1}-i \frac{c}{2}} \prod_{\delta=1}^{N^{e}} \frac{\chi_{\gamma}^{1}-1+i f \frac{c}{2}}{\chi_{\gamma}^{1}-1-i f \frac{c}{2}} \\
& \times \prod_{\beta=1}^{M^{2}} \frac{\chi_{\gamma}^{1}-\chi_{\beta}^{2}+i \frac{c}{2}}{\chi_{\gamma}^{1}-\chi_{\beta}^{2}-i \frac{c}{2}},
\end{aligned}
$$

and the energy is given by

$$
E=\sum_{\delta=1}^{N^{e} / f} f p_{\delta} .
$$

We proceed now to discuss the solutions of the fused equations. The solutions for the rank $r$ spin variables $\left\{\chi^{r}\right\}$ again fall into strings of arbitrary length $n$,

$$
\chi_{\gamma, j}^{r, n}=\chi_{\gamma}^{r, n}+i \frac{c}{2}(n+1-2 j) ; j=1, \ldots, n, n=1, \ldots, \infty .
$$

and a state is characterized by the quantum numbers $M^{r, m}$ specifying the number of length- $m$ strings of rank $r,\left(\sum_{m=1}^{\infty} m M^{r, m}=M^{r}\right)$.

The equations coupling the real part of the strings, after summing over the complex variables, can be conveniently written down in a logarithmic form. Let us first introduce the following definitions:

$$
\begin{aligned}
\theta_{n}(x) & \equiv-2 \arctan \left(\frac{2}{n c} x\right), \\
\phi_{n, m}^{k}(x) & \equiv \sum_{j=1}^{\min (n, m)} \theta_{m+n+k-2 j}(x), \\
\phi_{n, m}^{0}(x) & \equiv \sum_{j=1}^{\min (n-1, m-1)} \theta_{m+n-2 j}(x) .
\end{aligned}
$$


Then, after some manipulations the Bethe-Ansatz equations take the form,

$$
\begin{aligned}
& \sum_{m=1}^{\infty} \sum_{\beta=1}^{M^{r, m}}\left(\phi_{n, m}^{2}\left(\chi_{\gamma}^{r, n}-\chi_{\beta}^{r, m}\right)+\phi_{n, m}^{0}\left(\chi_{\gamma}^{r, n}-\chi_{\beta}^{r, m}\right)\right) \\
= & 2 \pi I_{\gamma}^{r, n}+\sum_{l=1}^{\infty} \sum_{\beta=1}^{M^{r-1, l}} \phi_{n, l}^{1}\left(\chi_{\gamma}^{r, n}-\chi_{\beta}^{r-1, l}\right) \\
+ & \sum_{l=1}^{\infty} \sum_{\beta=1}^{M^{r+1, l}} \phi_{n, l}^{1}\left(\chi_{\gamma}^{r, n}-\chi_{\beta}^{r+1, l}\right), \\
& \sum_{m=1}^{\infty} \sum_{\beta=1}^{M^{1, m}}\left(\phi_{n, m}^{2}\left(\chi_{\gamma}^{1, n}-\chi_{\beta}^{1, m}\right)+\phi_{n, m}^{0}\left(\chi_{\gamma}^{1, n}-\chi_{\beta}^{1, m}\right)\right) \\
= & 2 \pi I_{\gamma}^{1, n}+\phi_{n, 1}^{1}\left(\chi_{\gamma}^{1, n}\right)+\frac{N^{e}}{f} \phi_{n, f}^{1}\left(\chi_{\gamma}^{1, n}-1\right) \\
+ & \sum_{l=1}^{\infty} \sum_{\beta=1}^{M^{2, l}} \phi_{n, l}^{1}\left(\chi_{\gamma}^{1, n}-\chi_{\beta}^{2, l}\right) .
\end{aligned}
$$

The expression for the energy of the spin and charge sector is given by

$$
\begin{aligned}
E & =\sum_{\delta=1}^{N^{e} / f} \frac{2 \pi}{L} m_{\delta} \\
& +\frac{D}{f} \sum_{n=1}^{\infty} \sum_{\beta=1}^{M^{1, n}}\left(\phi_{n, f}^{1}\left(\chi_{\gamma}^{1, n}-1\right)-\pi \min (n, f)\right)
\end{aligned}
$$

where $D=\frac{N^{e}}{L}$ is the electron density. It will turn out also to play the role of the cut-off. In the presence of a magnetic field, $H$, there is a contribution to the energy of the form,

$$
\begin{aligned}
-2 H \sum_{k=0}^{N-1} & \left(M^{k}-M^{k+1}\right)\left(\frac{N-1}{2}-k\right)= \\
& =-2 H\left(\frac{N-1}{2}\left(N^{e}+1\right)-\sum_{r=1}^{N} M^{r}\right) .
\end{aligned}
$$

We now take the thermodynamic limit, $N^{e} \rightarrow \infty$, $L \rightarrow \infty$, holding $D$ finite. In the limit we may replace sums with integrals after introducing densities of solutions, $\sigma_{n}^{r}(\chi)$, and densities of holes in the distribution of solutions, $\sigma_{n}^{r, h}(\chi)$. The energy is now written as

$$
\begin{aligned}
E & =E_{c}-H(N-1)\left(N^{e}+1\right) \\
& +\sum_{n=1}^{\infty} \sum_{r=1}^{N-1} \int_{-\infty}^{\infty} d \chi \sigma_{n}^{r}(\chi) g_{r, n}(\chi),
\end{aligned}
$$

where we introduced the energy function,

$$
g_{r, n}(\chi)=\frac{D}{f}\left(\phi_{n, f}^{1}(\chi-1)-\pi \min (n, f)\right) \delta_{r, 1}+2 H n,
$$

and $E_{c}$ denotes the contribution of the charge sector to the energy,

$$
E_{c}=\sum_{\delta=1}^{N^{e} / f} \frac{2 \pi}{L} m_{\delta}
$$

In the thermodynamic limit the BAE are replaced by integral equations for the densities, $\left\{\sigma_{n}^{r}, \sigma_{n}^{r, h}\right\}$. Standard manipulations 10 lead to,

$$
\sigma_{n}^{r, h}(\chi)=-\sum_{m=1}^{\infty} \sum_{s=0}^{N} \int_{-\infty}^{\infty} d \chi^{\prime} A_{n, m}^{r, s}\left(\chi-\chi^{\prime}\right) \sigma_{m}^{s}\left(\chi^{\prime}\right) .
$$

where we introduced the following operators:

$$
\begin{aligned}
K_{n, m}^{\alpha}(\chi) & \equiv \sum_{j=1}^{\min (n, m)} k_{m+n+\alpha-2 j}(\chi), \\
\tilde{K}_{n, m}^{0}(\chi) & \equiv \sum_{j=1}^{\min (n-1, m-1)} k_{m+n-2 j}(\chi), \\
k_{\alpha}(\chi) & \equiv-\frac{1}{2 \pi} \theta_{\alpha}(\chi), \quad k_{0}(\chi) \equiv \delta(\chi) .
\end{aligned}
$$

and

$$
\begin{aligned}
& A_{n, m}(\chi) \equiv K_{n, m}^{2}(\chi)+K_{n, m}^{0}(\chi), \\
& B_{n, m}(\chi) \equiv K_{n, m}^{1}(\chi) \\
& A_{n, m}^{r, s} \equiv A_{n, m} \delta^{r, s}-B_{n, m}\left(\delta^{r, s+1}+\delta^{r, s-1}\right), \quad r \geq 1,
\end{aligned}
$$

and by convention,

$$
\begin{aligned}
& \sigma_{l}^{0}(\chi) \equiv \delta(\chi) \delta_{l, 1}+\frac{N^{e}}{f} \delta(\chi+1) \delta_{l, f}, \\
& \sigma_{n}^{N}(\chi) \equiv 0
\end{aligned}
$$

We shall not analyze here the ground state and the individual excitations. Instead, we shall proceed to derive the thermodynamic properties of the model.

\section{THERMODYNAMICS}

\section{A. Thermodynamic Bethe Ansatz equations}

We now calculate the impurity contribution to the free energy using the well known formalism of Yang and Yang 8 . We seek to find the configuration $\left\{\sigma_{n}^{r}(\chi)+\right.$ $\left.\sigma_{n}^{r, h}(\chi)\right\}$ which would extremize the free energy. The entropy of such a configuration is,

$$
\begin{aligned}
\mathcal{S}=\sum_{n, r} \int d \chi & \left\{\left(\sigma_{n}^{r}(\chi)+\sigma_{n}^{r, h}(\chi)\right) \ln \left(\sigma_{n}^{r}(\chi)+\sigma_{n}^{r, h}(\chi)\right)\right. \\
& \left.-\sigma_{n}^{r}(\chi) \ln \sigma_{n}^{r}(\chi)-\sigma_{n}^{r, h}(\chi) \ln \sigma_{n}^{r, h}(\chi)\right\}
\end{aligned}
$$

and its contribution to the spin free energy 


$$
\begin{aligned}
F= & E-T \mathcal{S}= \\
& -H(N-1)\left(N^{e}+1\right)+\sum_{n, r} \int d \chi\left\{\sigma_{n}^{r}(\chi) g_{r, n}(\chi)\right. \\
& -T\left(\left(\sigma_{n}^{r}(\chi)+\sigma_{n}^{r, h}(\chi)\right) \ln \left(\sigma_{n}^{r}(\chi)+\sigma_{n}^{r, h}(\chi)\right)\right. \\
& \left.\left.-\sigma_{n}^{r}(\chi) \ln \sigma_{n}^{r}(\chi)-\sigma_{n}^{r, h}(\chi) \ln \sigma_{n}^{r, h}(\chi)\right)\right\} .
\end{aligned}
$$

The free energy is varied with respect to the densities, subject to constraints imposed by the Bethe-Ansatz equations,

$$
\begin{aligned}
\delta \sigma_{n}^{r, h}(\chi) & =-\sum_{m, s} \int_{-\infty}^{\infty} d \chi^{\prime} A_{n, m}^{r, s}\left(\chi-\chi^{\prime}\right) \delta \sigma_{m}^{s}\left(\chi^{\prime}\right), \\
\delta \sigma_{n}^{0}(\chi) & =\delta \sigma_{n}^{N}(\chi)=0 .
\end{aligned}
$$

We obtain the following infinite set of integral equations for the equilibrium densities,

$$
\begin{aligned}
& \ln \left(1+\eta_{n}^{r}(\chi)\right)= \\
& \frac{g_{r, n}(\chi)}{T}+\sum_{m, s} \int_{-\infty}^{\infty} d \chi^{\prime} A_{n, m}^{r, s}\left(\chi-\chi^{\prime}\right) \ln \left(1+\left(\eta_{m}^{s}\left(\chi^{\prime}\right)\right)^{-1}\right),
\end{aligned}
$$

where

$$
\eta_{n}^{r}(\chi) \equiv \frac{\sigma_{n}^{r, h}(\chi)}{\sigma_{n}^{r}(\chi)}, \quad\left(\eta_{n}^{N}\right)^{-1} \equiv\left(\eta_{n}^{0}\right)^{-1} \equiv 0 .
$$

We transform this set of equations with the help of the following identities (we will now drop the functional dependence)

$$
\begin{aligned}
& A_{n, m}^{r, s}-G\left(A_{n-1, m}^{r, s}+A_{n+1, m}^{r, s}\right) \\
= & \delta_{n, m} \delta^{r, s}-G \delta_{n, m}\left(\delta^{r, s+1}+\delta^{r, s-1}\right) \\
& \quad A_{1, m}^{r, s}-G A_{2, m}^{r, s}=\delta_{1, m} \delta^{r, s}-G \delta_{1, m}\left(\delta^{r, s+1}+\delta^{r, s-1}\right),
\end{aligned}
$$

with the integral operator $G$ defined as

$$
G f(\chi) \equiv \frac{[1]}{[0]+[2]} f(\chi) \equiv \frac{1}{2 c} \int_{-\infty}^{\infty} d \chi^{\prime} \frac{f\left(\chi^{\prime}\right)}{\cosh \left(\frac{\pi}{c}\left(\chi-\chi^{\prime}\right)\right)},
$$

and $[n] f(\chi) \equiv \int_{-\infty}^{\infty} d \chi^{\prime} k_{n}\left(\chi-\chi^{\prime}\right) f\left(\chi^{\prime}\right)$. We find,

$$
\begin{aligned}
\ln \eta_{1}^{r}= & -\frac{2}{f} \frac{D}{T} \arctan e^{\frac{\pi}{c}(\chi-1)} \delta^{r, 1} \delta_{n, f}+G \ln \left(1+\eta_{1}^{r}\right) \\
& -G\left(\ln \left(1+\left(\eta_{1}^{r-1}\right)^{-1}\right)+\ln \left(1+\left(\eta_{1}^{r+1}\right)^{-1}\right)\right), \\
\ln \eta_{n}^{r}= & -2 \frac{D}{T f} \arctan e^{\frac{\pi}{c}(\chi-1)} \delta^{r, 1} \delta_{n, f} \\
& +G\left(\ln \left(1+\eta_{n-1}^{r}\right)+\ln \left(1+\eta_{n+1}^{r}\right)\right) \\
& -G\left(\ln \left(1+\left(\eta_{n}^{r-1}\right)^{-1}\right)+\ln \left(1+\left(\eta_{n}^{r+1}\right)^{-1}\right)\right),
\end{aligned}
$$

with boundary conditions,

$$
\lim _{n \rightarrow \infty}\left([n+1] \ln \left(1+\eta_{n}^{r}\right)-[n] \ln \left(1+\eta_{n+1}^{r}\right)\right)=-2 \frac{H}{T} .
$$

which follow directly from eqns (3.1). Another form of these equation can be obtained after inverting, see Ref. [ 8),

$$
\begin{aligned}
& -\ln \left(1+\left(\eta_{n}^{r}\right)^{-1}\right)=-\frac{\Delta E_{N, r}^{\text {fund }}}{T} \delta_{n, f} \\
& +\sum_{q=1}^{N-1} G_{N}^{r, q}\left(\ln \left(1+\eta_{n+1}^{q}\right)+\ln \left(1+\eta_{n-1}^{q}\right)\right. \\
& \left.-G^{-1} \ln \left(1+\eta_{n}^{q}\right)\right)
\end{aligned}
$$

where $\ln \left(1+\eta_{0}^{r}\right) \equiv 0$, and the Fourier transform of the kernel of the integral operator $G_{N}^{r, q}$ is given by

$$
\widetilde{G}_{N}^{r, q}(p) \equiv \frac{\sinh \left(\min (r, q) \frac{c p}{2}\right) \sinh \left((N-\max (r, q)) \frac{c p}{2}\right)}{\sinh \left(\frac{c p}{2}\right) \sinh \left(N \frac{c p}{2}\right)} .
$$

The driving term in these equations,

$$
\Delta E_{N, r}^{f \text { und }}=G_{N}^{r, 1} G^{-1}\left(2 \frac{D}{f} \arctan e^{\frac{\pi}{c}(\chi-1)}\right)
$$

is the energy of the fundamental excitation. It can be calculated explicitly,

$$
\begin{gathered}
\Delta E_{N, r}^{\text {fund }}=\frac{D}{f}\left\{\pi \frac{N-r}{N}\right. \\
\left.-2 \arctan \left(\tan \left(\frac{\pi}{2} \frac{N-r}{N}\right) \tanh \left(\frac{\pi}{N c}(\chi-1)\right)\right)\right\} .
\end{gathered}
$$
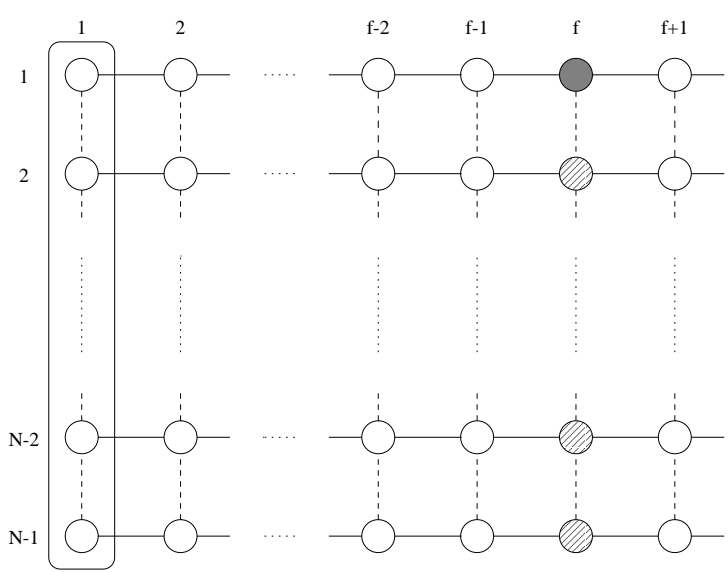

FIG. 1. Diagrammatic representation of the integral equations. The circles correspond to the functions $\eta_{n}^{r}$. The filled circle indicates that the equation for the corresponding $\eta_{n}^{r}$ has a driving term. The circles with stripes indicate that the corresponding $\eta_{n}^{r}$ have driving terms in the other set of TBA equations. The solid line indicates a link between two $\eta_{n}^{r}$ thorough the convolution $G \ln (1+\eta)$. The dashed line indicates a link through $G \ln (1+1 / \eta)$. Finally, the box encircles the $\eta_{1}^{r}$, which are the functions used to evaluate the impurity contribution to the free energy.

A pictorial description of (3.5) is shown in Fig. 1 . The circles correspond to the functions $\eta_{n}^{r}(x)$, and are arranged according to their indices. The lines join functions that appear in the same equation. The full and 
dotted lines indicate that the functions $\eta_{n}^{r \pm 1}$ and $\eta_{n \pm 1}^{r}$ appear differently in eqns (3.2,3.3). The driving terms in (3.2.3.3) correspond to filled dark circles in Fig. 1 . The diagonally lined circles correspond to functions associated to a driving term in (3.5). Clearly there are two regions: the one, $n<f$, contains a finite number of functions $\eta_{n}^{r}$, while the other, $n>f$, is unbounded. The regions are separated by the column with the driving terms, $n=f$. When studying the low-T properties of the system we will only need to consider one region at a time.

We will now write the free energy in terms of the set $\left\{\eta_{n}^{r}\right\}$. Using the integral equations for the densities we can write

$$
\begin{aligned}
F & =F_{0}+\sum_{n, r} \int d \chi\left(g_{r, n} \sigma_{n}^{r}-T \sigma_{n}^{r} \ln \left(1+\eta_{n}^{r}\right)\right. \\
& \left.+T \sum_{m, s} \int d \chi^{\prime} A_{n, m}^{r, s} \sigma_{n}^{r} \ln \left(1+\left(\eta_{m}^{s}\right)^{-1}\right)\right)
\end{aligned}
$$

where $F_{0}=E_{c}-H(N-1)\left(N^{e}+1\right)$ is the ground state energy. After a few further manipulations the free energy can be written as,

$$
\begin{aligned}
F= & F_{0}-T \sum_{n} \int_{-\infty}^{\infty} d \chi \ln \left(1+\left(\eta_{n}^{1}\right)^{-1}\right) \times \\
& \times\left\{k_{n}(\chi)+\frac{N^{e}}{f} \sum_{j=1}^{\min (n, f)} k_{f+n+1-2 j}(\chi-1)\right\} .
\end{aligned}
$$

We are only interested in the impurity contribution to the free energy, $F^{i}$, which contains all the effects of the interaction. It is

$$
\begin{aligned}
F^{i} & =-T \sum_{n=1}^{\infty} \int_{-\infty}^{\infty} d \chi \delta(\chi)[n] \ln \left(1+\left(\eta_{n}^{1}\right)^{-1}\right) \\
& =\sum_{q=1}^{N-1} \int_{-\infty}^{\infty} d \chi G_{N}^{1, q}(\chi) g_{1}^{q}(\chi) \\
& -T \sum_{q=1}^{N-1} \int_{-\infty}^{\infty} d \chi G_{N}^{1, q}(\chi) \ln \left(1+\eta_{1}^{q}\right),
\end{aligned}
$$

The first term corresponds to the impurity contribution to the ground state. At finite temperatures we are only interested in the second term which after further manipulations becomes,

$F^{i}=-T \sum_{q=1}^{N-1} \int_{-\infty}^{\infty} d \chi \frac{1}{N c} \frac{\sin \pi \frac{N-q}{N}}{\cosh \frac{2 \pi \chi}{N c}+\cos \pi \frac{N-q}{N}} \ln \left(1+\eta_{1}^{q}(\chi)\right)$.

When the impurity is in the fundamental representation, only the $\eta_{1}^{r}$ functions contribute to $F^{i}$. In Fig. 1 this feature corresponds to a box drawn around the first column.

\section{B. Scaling limit}

We will now take the scaling limit, $D \rightarrow \infty, c \rightarrow 0, T_{0}$ constant, where

$$
T_{0}=D e^{-\frac{2 \pi}{N c}}
$$

This is the correct limit as discussed in Ref. [8]. We also introduce the new variable

$$
\xi=\frac{2 \pi}{N c} \chi+\ln \frac{T_{0}}{T}
$$

so

$$
F^{i}=-\frac{T}{2 \pi} \sum_{q=1}^{N-1} \int_{-\infty}^{\infty} d \xi \frac{\left(\sin \pi \frac{N-q}{N}\right) \ln \left(1+\eta_{1}^{q}(\xi)\right)}{\cosh \left(\xi-\ln \frac{T_{0}}{T}\right)+\cos \pi \frac{N-q}{N}}
$$

The only modification in the thermodynamic equations is in $\Delta E_{N, r}^{\text {fund }}$. Thus

$$
\begin{aligned}
& -\ln \left(1+\left(\eta_{n}^{r}\right)^{-1}\right)=-\frac{2}{f} e^{\xi} \sin \left(\frac{\pi r}{N}\right) \delta_{n, f} \\
& +\sum_{q=1}^{N-1} G_{N}^{r, q}\left(\ln \left(1+\eta_{n+1}^{q}\right)+\ln \left(1+\eta_{n-1}^{q}\right)\right. \\
& \left.-G^{-1} \ln \left(1+\eta_{n}^{q}\right)\right), \\
\eta_{0}^{r} & \equiv 0, \ln \left(1+\left(\eta_{n}^{0}\right)^{-1}\right) \equiv 0, \ln \left(1+\left(\eta_{n}^{f}\right)^{-1}\right) \equiv 0,
\end{aligned}
$$

with boundary conditions

$$
\lim _{n \rightarrow \infty}\left([n+1] \ln \left(1+\eta_{n}^{r}\right)-[n] \ln \left(1+\eta_{n+1}^{r}\right)\right)=-2 \frac{H}{T} .
$$

\section{Asymptotic solutions-Low temperature properties}

Here we will study several asymptotic limits of the thermodynamic integral equations. Some technical points will be considered in detail. It is not easy to study analytically the integral equations 3.7 3.9 due to the complexity of the operator $G_{N}^{r, q}$. Instead, we will study the equivalent set equations (3.2 3.4). We will discuss the appropriate procedure to obtain the asymptotic solutions of the equations order by order.

The zeroth order approximation yields a description of the fixed point itself, the corrections (first order) describe its neighborhood.

\section{Zeroth order - the fixed point}

The functions $\eta_{n}^{r}$ tend either to 0 or to constant values as the magnitudes of their arguments tend to infinity. The only information needed about the driving term is that it tends to 0 as $\xi \rightarrow-\infty$, and to $-\infty$ as 
$\xi \rightarrow \infty$. Therefore, the parameter $\chi$ does not appear explicitly in (3.2 3.4 ), and the kernel of $G$ can be replaced by $(1 / 2) \delta\left(\xi-\xi^{\prime}\right)$.

Thus, the zeroth order problem consists in evaluating the following set of constants

$$
\eta_{n}^{r, \pm} \equiv \eta_{n}^{r}(\xi \rightarrow \pm \infty)
$$

When $\xi \rightarrow-\infty$, all the driving terms vanish, and the algebraic equations for the set $\left\{\eta_{n}^{r,-}\right\}$ are

$$
\begin{aligned}
& 2 \ln \eta_{n}^{r,-}=\ln \left(1+\eta_{n+1}^{r,-}\right)+\ln \left(1+\eta_{n-1}^{r,-}\right) \\
& +\ln \left(1+\left(\eta_{n}^{r+1,-}\right)^{-1}\right)+\ln \left(1+\left(\eta_{n}^{r-1,-}\right)^{-1}\right), \\
& \eta_{0}^{r,-}=0 \text {, } \\
& \ln \left(1+\left(\eta_{n}^{0,-}\right)^{-1}\right)=\ln \left(1+\left(\eta_{n}^{N,-}\right)^{-1}\right)=0, \\
& -2 \frac{H}{T}=\lim _{n \rightarrow \infty}\left([n+1] \ln \left(1+\eta_{n}^{r,-}\right)-[n] \ln \left(1+\eta_{n+1}^{r,-}\right)\right),
\end{aligned}
$$

Since the kernel of $G_{N}^{r, q}(\chi)$ satisfies

$$
G_{N}^{r, q}=G_{N}^{q, r}, \quad G_{N}^{r, q}=G_{N}^{N-r, N-q}
$$

we must have $\eta_{n}^{N-r}=\eta_{n}^{r}$. The solution is easily obtained as it is independent of the flavor symmetry,

$$
\begin{gathered}
\eta_{n}^{r,-}=\frac{\sinh \left((n+r) x_{0}\right) \sinh \left((n+N-r) x_{0}\right)}{\sinh \left(r x_{0}\right) \sinh \left((N-r) x_{0}\right)}-1 \\
n=1,2, \ldots, \quad r=1, \ldots, N-1, \quad x_{0}=\frac{H}{T}
\end{gathered}
$$

As for $\eta_{n}^{n,+}$, we should consider separately the cases $n>f$ and $n<f\left(\eta_{f}^{r,+}=0\right)$. In the first case, we have equations similar to those for $\left\{\eta_{n}^{r,-}\right\}$, except that all the indices $n$ are shifted by $f$, in analogy with the multichannel Kondo model. Hence

$$
\begin{gathered}
\eta_{n}^{r,+}=\frac{\sinh \left((n-f+r) x_{0}\right) \sinh \left((n-f+N-r) x_{0}\right)}{\sinh \left(r x_{0}\right) \sinh \left((N-r) x_{0}\right)}-1, \\
n=f, f+1, \ldots, \quad r=1, \ldots, N-1 .
\end{gathered}
$$

Finally, for $n<f$ there are a finite number of $\eta_{n}^{r,+}$ involved, since $\eta_{f}^{r,+}=0$, and $G \ln \left(1+\eta_{f}^{r}\right)=0$. As in the multichannel Kondo 6 , the sinh functions are replaced by sin functions, and the coefficients are independent of the magnetic field. Thus

$$
\begin{gathered}
\eta_{n}^{r,+}=\frac{\sin \left(\frac{\pi}{(f+N)}(n+r)\right) \sin \left(\frac{\pi}{(f+N)}(n+N-r)\right)}{\sin \left(\frac{\pi}{(f+N)} r\right) \sin \left(\frac{\pi}{(f+N)}(N-r)\right)}-1 \\
n=1, \ldots, f-1 \quad r=1, \ldots, N-1
\end{gathered}
$$

These results coincide with those obtained in Ref. [15] for a model of interacting fermions with the same symmetry. This is not surprising since these results depend only on the symmetry of the problem. Notice that the multichannel Kondo results correspond to $3.10-3.12$ with $N=2$. Some features of $(3.10-3.12)$ can be appreciated in Fig. 1. When $\xi \rightarrow-\infty$ the driving term does not contribute to the equations and the situation is the same as in the Coqblin-Schrieffer model. For $\xi \rightarrow \infty$, and $n \geq f$, we can disregard the $n<f$ sector, and the leftover diagram is effectively the same as for the Coqblin-Schrieffer model with the substitution $n \rightarrow n-f$. Finally, for $n<f$ we have a finite number of $\eta_{n}^{r,+}$ involved. Hence the replacement of the sinh by $\sin$.

\section{Residual entropy - the fixed point}

Here we will calculate the residual entropy in the overscreened case, $f>1$. As $T \rightarrow 0$, the dominant term in the free energy will be linear, and it will depend only on the values of $\eta_{1}^{r,+}$.

$$
\begin{aligned}
F^{i} & \sim-\frac{T}{2 \pi} \sum_{q=1}^{N-1} \sin \left(\pi \frac{N-q}{N}\right) \\
& \times \int_{0}^{\infty} d \xi \frac{\ln \left(1+\eta_{1}^{q,+}\right)}{\cosh \left(\xi-\ln \frac{T_{0}}{T}\right)+\cos \left(\pi \frac{N-q}{N}\right)} \\
& \sim-T \sum_{q=1}^{N-1}\left(\frac{N-q}{N}\right) \ln \left(1+\eta_{1}^{q,+}\right),
\end{aligned}
$$

Substituting the values of $\eta_{1}^{q,+}$, and taking advantage of the symmetry that $\eta_{1}^{N-q,+}=\eta_{1}^{q,+}$, we find

$$
F^{i}=-T \ln \frac{\sin \frac{\pi N}{f+N}}{\sin \frac{\pi}{f+N}}
$$

Hence, the residual entropy is

$$
S_{T=0}^{i}=-\left.\frac{\partial F^{i}}{\partial T}\right|_{T=0}=\ln \frac{\sin \frac{\pi N}{f+N}}{\sin \frac{\pi}{f+N}}=\ln \frac{\sin \frac{\pi f}{f+N}}{\sin \frac{\pi}{f+N}}
$$

Once again, we recover the multichannel results if we set $N=2$. It is quite clear that it is not the logarithm of an integer number!

The expression for the entropy can be written as the sum of two terms: one that depends only on $N+f$ and a second one that depends only on $|\log \gamma|,(\gamma=f / N)$

$$
S^{i}=\ln \sin \frac{\pi}{1+e^{|\log \gamma|}}-\ln \sin \frac{\pi}{N+f}
$$

In the limits $f \gg N$ and $f \ll N$ we have

$$
S^{i}=\left\{\begin{array}{cl}
\ln N-\frac{\pi^{2}}{6} \frac{N^{2}-1}{f^{2}}, & f \gg N, \\
\ln f-\frac{\pi^{2}}{6} \frac{f^{2}-1}{N^{2}}, & f \ll N .
\end{array}\right.
$$

Furthermore, it is clear from (3.17) that two systems characterized by $\gamma_{1}$ and $\gamma_{2}$ such that $\gamma_{1}=1 / \gamma_{2}$ have the same residual entropy. When $\gamma=1$, the first term is zero. 
Fig. 2 corresponds to (3.16) for different values of $N$ and $f$. It is quite apparent that the value of $S^{i}$ increases with $N+f$. It is also clear that the figure is symmetric with respect to the $N=f$ axis, which means that $S^{i}$ is the same for $\gamma$ and for $1 / \gamma$. Finally, if we fix $N+f$, the largest value of the residual entropy corresponds to $N=f$.

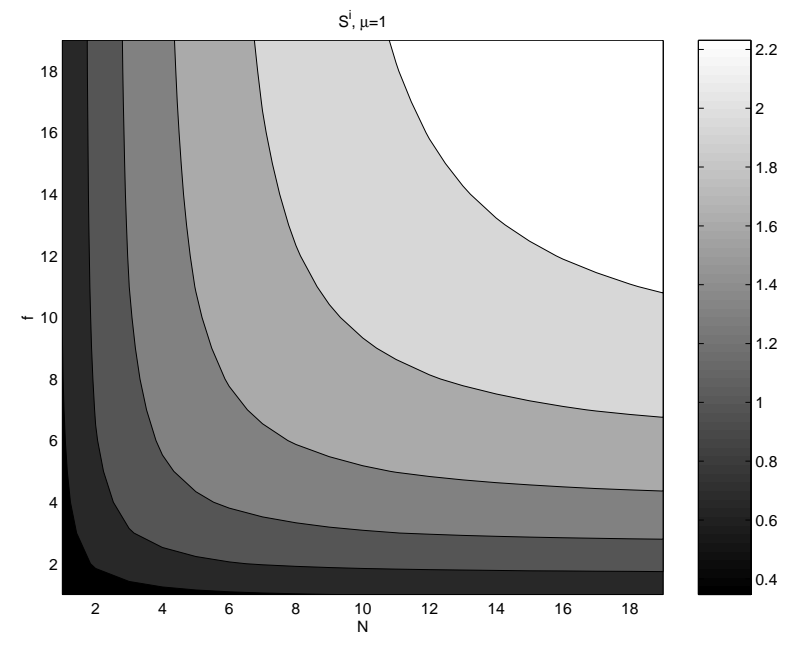

FIG. 2. Overscreening residual entropy, $S^{i}$, for an impurity in the fundamental representation of $S U(N)$, and for different values of $N$ and $f$.

In terms of the diagrammatic construction, Fig. 1, $S^{i}$ measures the size of the overscreened region and how asymmetric the region is. For fixed $N+f$, the largest residual entropy corresponds to $\gamma=1$, in the same way as the square is the rectangle with the largest area for a fixed perimeter. This can be seen in the following diagrams, were we have omitted the lines and drawn only the circles corresponding to $n<f$.

$$
N=7, f=3
$$

$N=f=5$
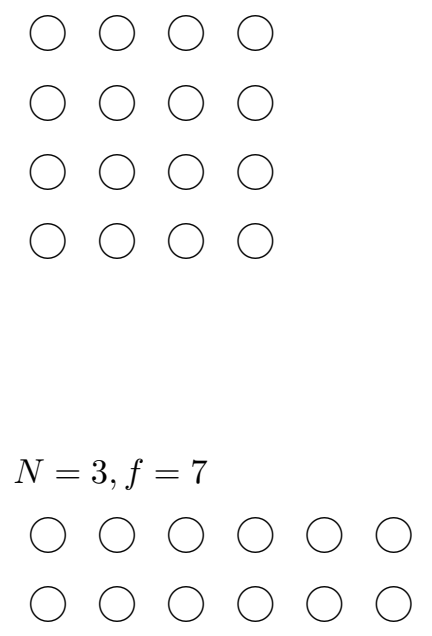

The largest entropy corresponds to the configuration with the largest number of circles, for a fixed value of $N+f$. That is, $N=f$. Notice that the first and the third cases have the same number of circles and, indeed, the same value of of the impurity entropy $S^{i}$.

\section{First order - the neighborhood of the fixed point}

Now we turn to the calculation of the thermodynamic properties of the $S U(N) \times S U(f)$ model well below the Kondo scale $T_{0}$. As is obvious from Eq. (3.14) for $T \ll T_{0}$ the nontrivial temperature dependence of the impurity free energy is determined by the asymptotic behavior of the functions $\eta_{1}^{q}(\xi)$ in the region $\xi \gg 1$. Therefore we want to find the dominant dependence of $\eta_{n}^{r}$ on $\xi$ for $n<f$ and $\xi$ large and positive. To this purpose we will only need the equations with $n<f$, which do not have a driving term, and the asymptotic value of $\eta_{f}^{r}$.

Consider the action of the operator $G$

$$
\begin{aligned}
G_{\chi} f & =\frac{1}{2 c} \int_{-\infty}^{\infty} d \chi^{\prime} \frac{f\left(\chi^{\prime}\right)}{\cosh \left(\frac{\pi}{c}\left(\chi-\chi^{\prime}\right)\right)} \\
& =\frac{1}{2 c} \int_{-\infty}^{\infty} d \chi^{\prime} \frac{\tilde{f}\left(\frac{2 \pi \chi^{\prime}}{N c}+\ln \frac{T_{0}}{T}\right)}{\cosh \left(\frac{N}{2} \frac{2 \pi}{N c}\left(\chi-\chi^{\prime}\right)\right)} \\
& =\frac{N}{4 \pi} \int_{-\infty}^{\infty} d \xi^{\prime} \frac{\tilde{f}\left(\xi^{\prime}\right)}{\cosh \left(\frac{N}{2}\left(\xi-\xi^{\prime}\right)\right)} \equiv G_{\xi} f
\end{aligned}
$$

where we have dropped the tilde in the last expression. This establishes the correspondence between functions and variables of the two systems of equations.

From the set of equations (3.2 3.4), and the asymptotic values (3.7-3.9) we learn that for large and positive values of $\xi$ we have (if $x_{0}=H / T$ is very small) 


$$
\eta_{f}^{r}(\xi) \propto\left(\alpha^{r}+\beta^{r} x_{0}^{2}\right) e^{-\frac{2}{f} \sin \left(\frac{\pi r}{N}\right) e^{\xi}}
$$

We now evaluate the dominant contribution to $G_{\xi} \ln (1+$ $\left.\eta_{f}^{r}\right)$

$$
\begin{aligned}
& G_{\xi} \ln \left(1+\eta_{f}^{r}\right)=\frac{N}{4 \pi} \int_{-\infty}^{\infty} d \xi^{\prime} \frac{\ln \left(1+\eta_{f}^{r}\right)}{\cosh \frac{N}{2}\left(\xi-\xi^{\prime}\right)} \\
& \xi \gtrsim^{2} \frac{N}{4 \pi}\left(\alpha^{r}+\beta^{r} x_{0}^{2}\right) \int_{\omega}^{\infty} d \xi^{\prime} \frac{e^{-\Delta e^{\xi^{\prime}}}}{\cosh \frac{N}{2} \xi^{\prime}},
\end{aligned}
$$

where $\omega$ is some lower cutoff of the integral of the order of unity and

$$
\Delta \equiv \frac{2}{f} \sin \left(\frac{\pi r}{N}\right) e^{\xi}
$$

For large $\xi$, the only relevant contribution to the integral occurs around $e^{\xi^{\prime}} \sim 1 / \Delta$. Therefore, we approximate the previous integral by

$$
\left(\alpha^{r}+\beta^{r} x_{0}^{2}\right) \frac{\Delta^{\frac{N}{2}}}{1+\Delta^{N}} \sim\left(\alpha^{r}+\beta^{r} x_{0}^{2}\right) e^{-\frac{N}{2} \xi} .
$$

Notice that this is correct up to terms of the form $\xi^{\mu} e^{-\frac{N}{2} \xi}$ which cannot be accounted for using this crude approximation.

The previous calculation indicates that $\eta_{n<f}^{r}$ will have a contribution of order $e^{-\frac{N}{2} \xi}$, since $G_{\xi} e^{-\alpha \xi} \propto e^{-\alpha \xi}$, as we will see below. Therefore, we have to determine whether there are contributions more singular still. In other words, we have to find out if there are solutions of the integral equations for large $\xi$ of the form

$$
\eta_{n<f}^{r}(\xi) \sim \eta_{n<f}^{r,+}+c_{n}^{r}\left(\alpha^{r}+\beta^{r} x_{0}^{2}\right) e^{-\tau \xi}, \text { with } \tau<\frac{N}{2} .
$$

Introducing the eigenvalue $\lambda \equiv 2 \cos \frac{\pi \tau}{N}$, so that

$$
G e^{-\tau \xi}=\frac{e^{-\tau \xi}}{\lambda}
$$

we proceed to convert the TBA equations into algebraic recursion relations. Noting that,

$$
\begin{aligned}
\ln \left(1+\eta_{n}^{r}\right) & \sim \ln \left(1+\eta_{n}^{r,+}\right)+\left(b_{n}^{r}+a_{n}^{r} x_{0}^{2}\right) e^{-\tau \xi} \\
\ln \eta_{n}^{r} & \sim \ln _{n}^{r,+}+\frac{\left(b_{n}^{r}+a_{n}^{r} x_{0}^{2}\right)}{\omega_{n}^{r}} e^{-\tau \xi}
\end{aligned}
$$

where,

$$
\begin{aligned}
\left(b_{n}^{r}+a_{n}^{r} x_{0}^{2}\right) & \equiv \frac{c_{n}^{r}\left(\alpha^{r}+\beta^{r} x_{0}^{2}\right)}{1+\eta_{n}^{r,+}} \\
\omega_{n}^{r} & \equiv \frac{\eta_{n}^{r,+}}{1+\eta_{n}^{r,+}}
\end{aligned}
$$

then substituting in the integral equations 3.2,3.3) for $n<f$ and using the zeroth-order results we obtain the following set of algebraic equations for the coefficients of $e^{-\tau \xi}$ (we only write the equations for $b_{n}^{r}$ since they are identical to those for $a_{n}^{r}$ ),

$$
\lambda b_{n}^{r}=\omega_{n}^{r}\left(b_{n+1}^{r}+b_{n-1}^{r}\right)+\frac{\omega_{n}^{r}}{\eta_{n}^{r+1,+}} b_{n}^{r+1}+\frac{\omega_{n}^{r}}{\eta_{n}^{r-1,+}} b_{n}^{r-1},
$$

with

$$
b_{0}^{r}=b_{f}^{r}=0 .
$$

More explicitly, upon inserting zero order values the equations become,

$$
\begin{aligned}
\lambda b_{n}^{r} & =\frac{\sin ((n+N) a) \sin (n a)}{\sin ((n+r) a) \sin ((n+N-r) a)}\left(b_{n+1}^{r}+b_{n-1}^{r}\right) \\
& +\frac{\sin ((r+1) a) \sin ((N-r-1) a)}{\sin ((n+r) a) \sin ((n+N-r) a)} b_{n}^{r+1} \\
& +\frac{\sin ((r-1) a) \sin ((N-r+1) a)}{\sin ((n+r) a) \sin ((n+N-r) a)} b_{n}^{r-1}
\end{aligned}
$$

where

$$
a \equiv \frac{\pi}{f+N}
$$

We solve (3.24) by inspection. Since $b_{n}^{r}$ has to satisfy the boundary conditions, (3.23), we have that

$$
b_{n}^{r}=\sin ((n+N) a) \sin (n a) d_{n}^{r}
$$

is the maximal solution when $d_{n}^{r}=d=$ constant, and the eigenvalue is,

$$
\lambda=2 \cos \frac{\pi \tau}{N}=2 \cos \frac{2 \pi}{f+N} .
$$

Hence, finally

$$
\tau=\frac{2 N}{N+f}
$$

We shall see next section that $\tau$ is the main critical exponent in the model.

\section{Specific heat and finite temperature susceptibility}

The expression for the impurity contribution to the free energy, $F^{i}$, has always at low temperatures a term which is proportional to $T^{2}$.

This contribution comes from the term proportional to $e^{-\frac{N}{2} \xi}$ present in $\eta_{n<f}^{r}$, as we discussed in the previous subsection.

Here, we will study contributions of the form

$$
\begin{aligned}
& \Delta F^{i} \propto-\frac{T}{2 \pi} \int_{-\infty}^{\infty} d \xi \sum_{q=1}^{N} \frac{\sin \left(\pi \frac{N-q}{N}\right)\left(\alpha^{q}+\beta^{q} x_{0}^{2}\right) e^{-\tau \xi}}{\cosh \left(\xi-\ln \frac{T_{0}}{T}\right)+\cos \pi \frac{N-q}{N}} \\
= & -\frac{T}{2 \pi}\left(\frac{T}{T_{0}}\right)^{\tau} \int_{-\infty}^{\infty} d \xi \sum_{q=1}^{N} \frac{\sin \left(\pi \frac{N-q}{N}\right)\left(\alpha^{q}+\beta^{q} x_{0}^{2}\right) e^{-\tau \xi}}{\cosh (\xi)+\cos \pi \frac{N-q}{N}},
\end{aligned}
$$


which might become dominant depending on the value of $\tau$. We will consider the three cases $f>N, f=N$, and $f<N$ separately.

Case $f>N$ : In this case, $\tau<1$, and

$$
\lim _{\xi \rightarrow-\infty} \frac{e^{-\tau \xi}}{\cosh \xi}=0
$$

That means that we can make the same the approximation for the free energy that we made when we evaluated $G_{\xi} \ln \left(1+\eta_{n}^{r}\right)$. Therefore, we have

$$
F^{i} \sim-T S^{i}-T\left(A+B\left(\frac{H}{T}\right)^{2}\right)\left(\frac{T}{T_{0}}\right)^{\tau}
$$

with $A$ and $B$ being constants o the order of unity and we obtain

$$
C^{i} \propto\left(\frac{T}{T_{0}}\right)^{\frac{2 N}{N+f}}, \quad \chi^{i} \propto \frac{1}{T_{0}}\left(\frac{T}{T_{0}}\right)^{\frac{N-f}{N+f}} .
$$

Needless to say, when $N=2$ we recover the multichannel results. As a matter of fact, the exponents depend only on the ratio $\gamma=f / N$, $1 \mathrm{E}$.

Case $f=N$ : Since $\tau=1$ in this case, we cannot extend the integral in eq. (3.27) to $\xi \rightarrow-\infty$ and we have to restrict it to the interval $[\delta, \infty)$, where $\delta$ is a finite number of the order of one. Making use of $\int \frac{d z}{1+e^{2 z}}=z-\frac{1}{2} \ln \left(1+e^{2 z}\right)$ we have for very low temperatures

$$
\begin{aligned}
\Delta F^{i} & \propto-\frac{T}{2 \pi}\left(\frac{T}{T_{0}}\right) \int_{\delta-\ln \frac{T_{0}}{T}}^{\infty} \sum_{q=1}^{N} \frac{\left(\sin \pi \frac{N-q}{N}\right)\left(\alpha^{q}+\beta^{q} x_{0}^{2}\right) e^{-\xi}}{\cosh (\xi)+\cos \pi \frac{N-q}{N}} \\
& \propto-T S^{i}+\frac{T^{2}}{T_{0}} \frac{\ln T}{T_{0}}\left(A+B\left(\frac{H}{T}\right)^{2}\right) .
\end{aligned}
$$

Hence

$$
C^{i} \propto-\frac{T}{T_{0}} \ln \frac{T}{T_{0}}, \quad \chi^{i} \propto-\frac{1}{T_{0}} \ln \frac{T}{T_{0}} .
$$

Case $f<N$ : In this region $1<\tau<2$.

Consider the integral

$$
\int_{\delta-\log \frac{T_{0}}{T}}^{\infty} \frac{e^{-(\tau-1) x} d x}{1+e^{2 x}}=\frac{1}{\tau-1} \int_{\beta\left(\frac{T}{T_{0}}\right)^{\tau-1}}^{\infty} \frac{d y}{y^{2}\left(1+y^{\frac{2}{\tau-1}}\right)}
$$

It is possible to find a primitive for $\frac{2}{\tau-1}$ integer; we have,

$$
\int \frac{d y}{y^{2}\left(1+y^{\frac{2}{\tau-1}}\right)}=-\frac{1}{y}+\left\{\begin{array}{l}
\frac{1}{2 n} \sum_{k=1}^{n} \cos \frac{\pi(2 n-1)(2 k-1)}{2 n} \ln \left(1-2 y \cos \frac{\pi(2 k-1)}{2 n}+y^{2}\right) \\
-\frac{1}{n} \sum_{k=1} \sin \frac{\pi(2 k-1)(2 n-1)}{2 n} \arctan \left(\frac{y-\cos \frac{\pi(2 k-1)}{2 n}}{\left.\sin \frac{\pi(2 k-1)}{2 n}\right),}\right. \\
\frac{2}{\tau-1}=2 n \\
\frac{\ln (1+y)}{2 n+1}+\frac{1}{2 n+1} \sum_{k=1}^{n} \cos \frac{\pi(2 n)(2 k-1)}{2 n+1} \ln \left(1-2 y \cos \frac{\pi(2 k-1)}{2 n+1}+y^{2}\right) \\
-\frac{2}{2 n+1} \sum_{k=1} \sin \frac{\pi(2 k-1)(2 n)}{2 n+1} \arctan \left(\frac{y-\cos \frac{\pi(2 k-1)}{2 n+1}}{\left.\sin \frac{\pi(2 k-1)}{2 n+1}\right),} \quad \frac{2}{\tau-1}=2 n+1\right.
\end{array}\right.
$$

As $T \rightarrow 0$, the leading terms are of the form

$$
\left(\frac{T}{T_{0}}\right)^{\tau-1}+c t n
$$


Hence,

$$
\Delta F^{i} \propto-\left(A+B\left(\frac{H}{T}\right)^{2}\right)\left(\frac{T^{2}}{T_{0}}-\operatorname{ctn} . T\left(\frac{T}{T_{0}}\right)^{\tau}\right)
$$

Since $\tau>1$, the dominant term in $\Delta F^{i}$ is of order $T^{2}$. As for the specific heat and susceptibility, we have

$$
C^{i} \propto \frac{T}{T_{0}}-A\left(\frac{T}{T_{0}}\right)^{\frac{2 N}{N+f}}, \quad \chi^{i} \propto \frac{1}{T_{0}}-B\left(\frac{T}{T_{0}}\right)^{\frac{N-f}{N+f}} .
$$

These results are valid for any $1<\tau<2$, as can be verified by numerical integration of (3.31), or by the numerical solution of the thermodynamic equations.

To summarize, there are three different kinds of behavior in the overscreened sector, depending on the value of the ratio $\gamma=f / N$. i) When $\gamma>1$, both $C^{i} / T$ and $\chi^{i}$ have power-law divergences as $T \rightarrow 0$. The behavior is similar to that of the multichannel Kondo model with $f>2$. Indeed, the exponents are the same, since they depend on $\gamma$ only. ii) For $\gamma=1$ there are logarithmic divergences as in the two channel Kondo model. iii) When $\gamma<1$, the values of $C^{i} / T$ and $\chi^{i}$ at $T=0$ are finite. Actually, it can be deduced from the numerical analysis that these constants are the same as in the corresponding completely screened cases ( $\mu=f$, as we will see later). However, the fixed point has Non-Fermi liquid behavior, as can be seen from the value of the residual entropy and from the subleading power-law terms.

One can relate the different kinds of behavior to the shape of the $n<f$ sector in Fig. 1 as can be seen in the diagrams 3.19 3.21). The square diagram corresponds to $\gamma=1$, whereas the horizontal(vertical) one corresponds

\section{Channel Anisotropy}

In this section we consider briefly the case when some of the couplings $J_{m}$ are different. From the study of the analogous problem in the multichannel Kondo model19, we conclude that up to $f$ different energy scales will appear in the problem depending on the pattern of symmetry breaking. The novelty here is that there might be a situation where $\gamma>1$ for an intermediate regime of temperatures, whereas for very low temperatures the behavior is characterized by an effective $\gamma$ smaller than 1.

Consider a system where the flavor symmetry is such that $p$ energy scales $T_{1}<T_{2}<\ldots<T_{p}$, are generated. Each scale $T_{j}$ is related to a driving term at the level $n=m_{j}$ in the TBA equations (3.7). We will assume for simplicity, that $m_{1}<m_{2}<\ldots<m_{p}=f$. If the largest flavor symmetry possible is $S U(f)$ there will always be a driving term at the level $n=f$.

Then, when the temperature is below any $T_{j}$, the thermodynamic properties are given by (3.16) and (3.29), where $\gamma=f / N$ is replaced by $\gamma_{e f f}=m_{1} / N$. As the temperature is increased, the behavior of the system when
$T_{j-1}<T<T_{j}$ corresponds to $\gamma_{e f f}=m_{j} / N$. Indeed, the value to the impurity contribution to the entropy will be close to $\mathcal{S}^{i}\left(m_{j}, N\right)$.

Flavor anisotropy is a relevant perturbation of the isotropic hamiltonian. In general, the system will flow away from the fixed point characterized by $f$, and $N$ to a new fixed point characterized by $m_{1}<f$, and $N$. From (3.16) we see that $\mathcal{S}^{i}$ is reduced in such flow $\left(\mathcal{S}^{i}(f, N)\right.$ is monotonous in both $f$ and $N$ ).

It is worth noticing that, once $m_{1}<N, \chi^{i}$ and $C^{i} / T$ become constant as $T \rightarrow 0$.

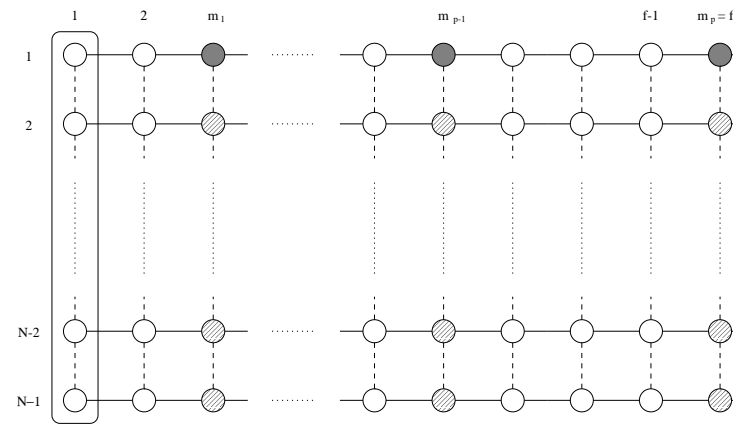

FIG. 3. Same as Fig. 1, but now the interaction with the impurity breaks the flavor symmetry from $S U(f)$ down to $\prod_{j=1}^{p} S U\left(m_{j}-m_{j-1}\right)$, with $m_{j}^{p}=f, m_{0}=0$.

The system of TBA equations are represented diagrammatically in Fig. 3. Depending on the pattern of symmetry breaking, driving terms appear at different values of $n$. There are always driving terms for $n=f$. The properties a temperatures between two different scales are related to the corresponding overscreened part of the diagram. Notice that channel anisotropy may have the effect of changing the shape in the overscreened area from something similar to (3.21) to diagrams like (3.20), and (3.21), but not the other way around.

\section{E. Impurity in a higher dimensional representation}

Finally, we study a generalization of the model in which the impurity behavior is that of an object in a rank $\mu$ representation of $S U(N)$. In the $S U(2)$ case it corresponds to an impurity with spin $S$. Following Ref. [ 20] and the same formulation that we followed for the fundamental representation, we find the following set of effective Bethe Ansatz equations

$$
\begin{gathered}
e^{i f p_{\delta} L}=\prod_{\gamma=1}^{M^{1}} \frac{\chi_{\gamma}^{1}-1+i f \frac{c}{2}}{\chi_{\gamma}^{1}-1-i f \frac{c}{2}}, \\
-\prod_{\beta=1}^{M^{r}} \frac{\chi_{\gamma}^{r}-\chi_{\beta}^{r}+i c}{\chi_{\gamma}^{r}-\chi_{\beta}^{r}-i c}=\prod_{\substack{t=r \pm 1\\
}} \prod_{\beta=1}^{M^{t}} \frac{\chi_{\gamma}^{r}-\chi_{\beta}^{t}+i \frac{c}{2}}{\chi_{\gamma}^{r}-\chi_{\beta}^{t}-i \frac{c}{2}} ; \\
r=2, \ldots, N-1,
\end{gathered}
$$




$$
\begin{aligned}
-\prod_{\beta=1}^{M^{1}} \frac{\chi_{\gamma}^{1}-\chi_{\beta}^{1}+i c}{\chi_{\gamma}^{1}-\chi_{\beta}^{1}-i c} & =\frac{\chi_{\gamma}^{1}+i \mu \frac{c}{2}}{\chi_{\gamma}^{1}-i \mu \frac{c}{2}} \prod_{\delta=1}^{N^{e} / f} \frac{\chi_{\gamma}^{1}-1+i f \frac{c}{2}}{\chi_{\gamma}^{1}-1-i f \frac{c}{2}} \\
& \times \prod_{\beta=1}^{M^{2}} \frac{\chi_{\gamma}^{1}-\chi_{\beta}^{2}+i \frac{c}{2}}{\chi_{\gamma}^{1}-\chi_{\beta}^{2}-i \frac{c}{2}}
\end{aligned}
$$
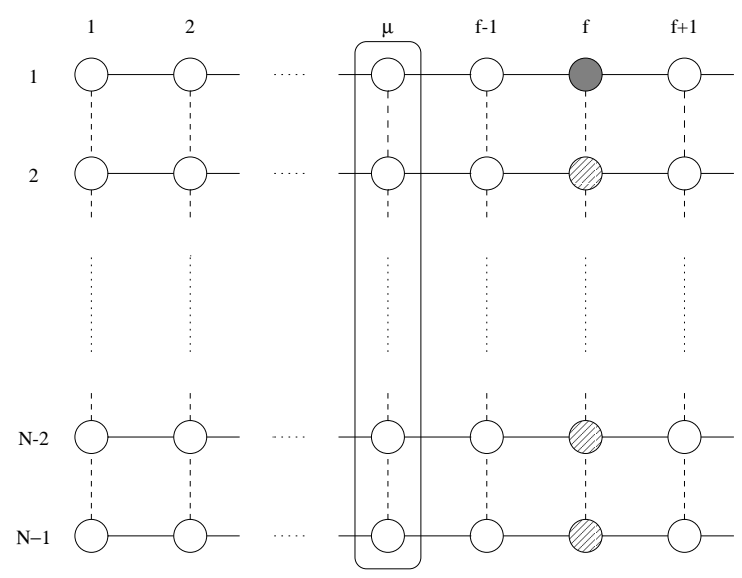

FIG. 4. Same as Fig. 1, but now the impurity contribution to the free energy involves the set $\eta_{\mu}^{q}$, where $\mu$ is the rank.

The impurity contribution to the free energy is

$$
F^{i}=-T \sum_{n} \int_{-\infty}^{\infty} B_{n, \mu} \ln \left(1+\left(\eta_{n}^{1}\right)^{-1}\right)
$$

As in the $\mu=1$, a series of transformations allow us to rewrite the free energy as

$$
\begin{aligned}
F^{i} & =\sum_{q=1}^{N-1} \int_{-\infty}^{\infty} d \chi G_{N}^{1, q}(\chi) g_{\mu}^{q}(\chi) \\
& -T \sum_{q=1}^{N-1} \int_{-\infty}^{\infty} d \chi G_{N}^{1, q}(\chi) \ln \left(1+\eta_{\mu}^{q}\right),
\end{aligned}
$$

where the first term corresponds to the impurity contribution to the ground state. At finite temperatures we are only interested in the second term, which in the scaling limit can be written as

$$
F^{i}=-\frac{T}{2 \pi} \sum_{q=1}^{N} \int_{-\infty}^{\infty} d \xi \frac{\left(\sin \pi \frac{N-q}{N}\right) \ln \left(1+\eta_{\mu}^{q}(\xi)\right)}{\cosh \left(\xi-\ln \frac{T_{0}}{T}\right)+\cos \pi \frac{N-q}{N}}
$$

The evaluation of $F^{i}$ involves the functions $\eta_{\mu}^{r}$, (see Fig. (4). The different scenarios possible are very similar to those of the multichannel Kondo model. As long as $\mu<f$, the impurity remains overscreened and the temperature exponents are the same as for the $\mu=1$ case. In this case, the residual entropy is

$$
\mathcal{S}_{T=0}^{i}=\ln \frac{\prod_{r=1}^{\mu+N-1} \sin \frac{\pi r}{f+N}}{\prod_{r=1}^{\mu} \sin \frac{\pi r}{f+N} \prod_{r=1}^{N-1} \sin \frac{\pi r}{f+N}}
$$

(Notice that when $N=2$ this reduces to the multichannel result $\left.\ln \frac{\sin \frac{\pi(2 S+1)}{f+2}}{\sin \frac{\pi}{f+2}}\right)$. Furthermore, it can be easily shown that $\mathcal{S}_{\mu}^{i}=\mathcal{S}_{f-\mu}^{i}$.

We have plotted $\mathcal{S}^{i}$ in Fig. 同, for fixed $N+f=22$, and several values of $\gamma=f / N$ and $\mu$. Only the region $\mu<f$ is physical in the figure, since these are results for the overscreened case. We can see that for fixed (even) $f$, the largest value of the entropy corresponds to $\mu=f / 2$. Also, for fixed $\mu$, the entropy is the largest around $\gamma \sim 1$, and decreases as $\gamma$ moves away from 1 .

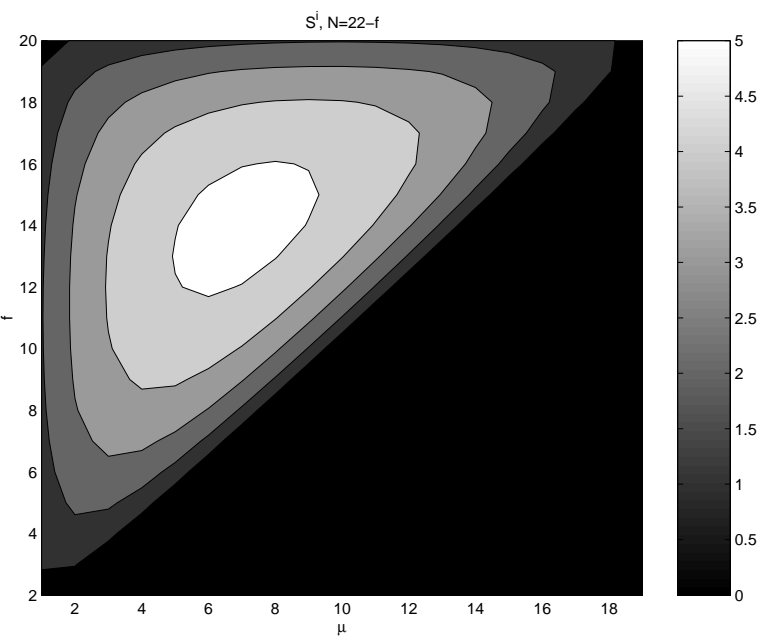

FIG. 5. Overscreening residual entropy, $S_{\mu<f}^{i}$, for an impurity in a totally symmetric representation, $\mu$, of $S U(N)$, for $N+f=22$. The lower triangular region (in black) is unphysical

If $\mu=f$ the impurity becomes completely screened: $\chi^{i}$ and $C^{i} / T$ become constant, and there is no residual entropy. Finally, if $\mu>f$, the impurity is underscreened. The dominant contribution to the free energy from the spin sector is of the form

$$
F^{i}=-T \ln \frac{\prod_{r=1}^{\mu-f+N-1} \sinh r \frac{H}{T}}{\prod_{r=1}^{\nu-f} \sinh r \frac{H}{T} \prod_{r=1}^{N-1} \sinh r \frac{H}{T}}
$$

When $H=0$, the residual entropy is

$$
\mathcal{S}^{i}=\ln \frac{(\mu-f+N-1) !}{(\mu-f) !(N-1) !}=\ln \left(\begin{array}{c}
\mu-f+N-1 \\
N-1
\end{array}\right)
$$

(For $N=2$, the residual entropy is $\ln \left(\begin{array}{c}\mu-f+1 \\ 1\end{array}\right)=$ $\ln (\mu-f+1))$.

\section{NUMERICAL ANALYSIS}




\section{A. Procedure}

We have solved the TBA equations by iterafion, using a procedure inspired by the work of Rajan21. For the levels $n \neq f$, which do not have a driving term (see Fig. (4), we have used (3.2,3.3), as the starting point, since it is more convenient to use the kernel $G(\xi)$. We have dealt with the equations that have a drifing term by introducing two sets of auxiliary functions 8 ,

$$
\begin{aligned}
& h^{r}(\xi)=G_{\xi}\left(\ln \left(1+\eta_{f+1}^{n}\right)+\ln \left(1+\eta_{f-1}^{n}\right)\right), \\
& Q^{r}(\xi)=G_{\xi}\left(Q^{r+1}\left(\xi^{\prime}\right)+Q^{r-1}\left(\xi^{\prime}\right)\right)-h^{r}(\xi),
\end{aligned}
$$

so that

$$
\ln \eta_{f}^{r}=-\frac{2}{f} e^{\xi} \sin \left(\frac{\pi r}{N}\right)+\ln \left(1+\eta_{f}^{r}\right)+Q^{r} .
$$

We have introduced a cutoff $A$ in the integrals involved, we have taken $\ln \left(1+\eta_{n}^{r}\right)$ to be constant for $|\xi|>A$, and evaluated the integrals in those intervals analytically. For $|\xi|<A$ we have replaced the integral with a sum using a Gaussian quadrature rule22.

The results that we present in this work correspond to zero magnetic field, which means that $x_{0}=H / T=0$, and the functions $\eta_{n}^{r}$ depend on $\xi$ only. Thus, the task of obtaining thermodynamic properties is greatly simplified. First of all, the impurity contribution is given by (3.6)

$$
F^{i}=-\frac{T}{2 \pi} \sum_{q=1}^{N} \int_{-\infty}^{\infty} d \xi \frac{\left(\sin \pi \frac{N-q}{N}\right) \ln \left(1+\eta_{\mu}^{q}(\xi)\right)}{\cosh \left(\xi-\ln \frac{T_{0}}{T}\right)+\cos \pi \frac{N-q}{N}}
$$

with $\eta_{n}^{r}$ independent of $T$. The entropy and specific heat are obtained by taking derivatives of $F^{i}$ with respect to the temperature, which can be done analytically when $x_{0}=0$, and then performing the integration numerically.

In order to calculate the susceptibility at zero magnetic field, $\chi^{i}$, we derived a second set of TBA equations for the functions

$$
\left.E_{n}^{r}(\xi) \equiv \frac{\partial^{2} \eta_{n}^{r}(\xi)}{\partial x_{0}^{2}}\right|_{x_{0}=0}
$$

following Degranges 23 . This system is solved as the previous one, and the magnetic susceptibility is given by

$$
\begin{aligned}
& \left.\chi^{i}\right|_{x_{0}=0}=\frac{\partial^{2} F^{i}}{\partial x_{0}^{2}}= \\
& -\frac{T}{2 \pi} \sum_{q=1}^{N} \int_{-\infty}^{\infty} d \xi \frac{\left(\sin \pi \frac{N-q}{N}\right) E_{\mu}^{q}(\xi)}{\cosh \left(\xi-\ln \frac{T_{0}}{T}\right)+\cos \pi \frac{N-q}{N}} .
\end{aligned}
$$

\section{B. Results}

\section{Entropy}

We start by discussing the impurity contribution to the entropy, $\mathcal{S}^{i}$. In Fig. 6 we have plotted $\mathcal{S}^{i}$ as a function of $T$, for different values of $N, f$, and impurity spin $\mu$. The horizontal axis is on a logarithmic scale. The vertical axes have different scales for the different $N$. The first thing to notice is the crossover around $T \sim T_{0}$. For $T \gg T_{0}$, $\mathcal{S}^{i}$ is that of a free spin characterized by $\mu$ and $N$. When $H=0$

$$
\mathcal{S}^{i}=\ln \left(\begin{array}{c}
\mu+N-1 \\
N-1
\end{array}\right)
$$

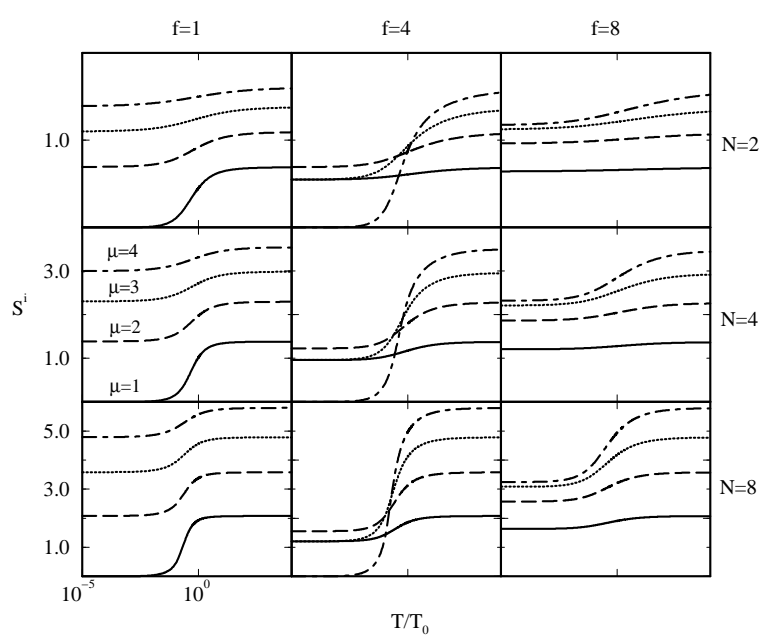

FIG. 6. Impurity contribution to the Entropy as a function of $T$, for different values of $N, f$, and impurity spin, $\mu$. Notice that different scales are used for the different $N$.

Below the crossover region one can see the quenching of the degrees of freedom due to the interaction in the decrease of the value of the entropy. The qualitative behavior of $\mathcal{S}^{i}$ in the region $T \ll T_{0}$ depends only on the relation between $\mu$ and $f$. When $f=\mu$, there is complete screening, and $\mathcal{S}^{i}=0$, as can be seen in the curves $\mu=f=1, \mu=f=4$. For $\mu>f$, the impurity is not completely screened, and there is effectively a leftover free spin $\mu-f$, as can be seen in Fig. 6 for $f=1$. There, the $T \ll T_{0}$ entropy for $\mu<f$ corresponds to the $T \gg T_{0}$ entropy for $\mu-1$. Finally, when $\mu<f$, overscreening takes place: even though there are enough electrons to form a singlet with the impurity, the low temperature behavior is characterized by an object with complex internal structure, and an anomalous $\mathcal{S}^{i}$. Such behavior can be seen in the curves $\mu=2, \mu=3$ for $f=4$, and in all the curves for $f=8$. Notice that for $\mu=1$ and $\mu=f-1$, the curves converge to the same value, as we had already seen in the asymptotic analysis. Furthermore, the overscreened fixed point has an anomalous 
residual entropy irrespective of the value of $N$, indicating its Non-Fermi liquid nature.

A more detailed picture of the behavior of $\mathcal{S}^{i}$ for $N=4$ is displayed in Fig. 7. It is worth noticing that in the underscreened cases, the effective spin is $\mu-f$. Also, there might be situations where the residual entropy of the overscreened case is larger than that of the underscreened case. Such is the case for $f=5, \mu=3,4$, as compared to $\mu=6$.

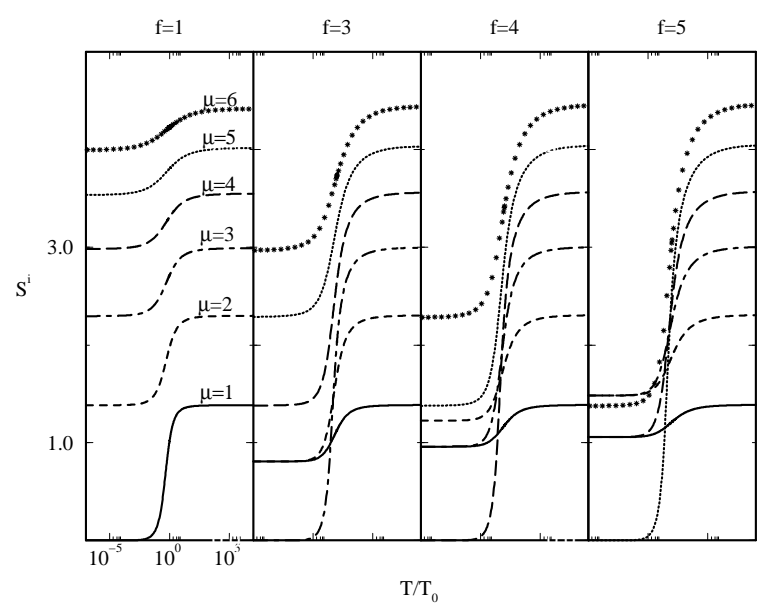

FIG. 7. $\mathcal{S}^{i}$ vs. $T$ for $N=4, f=1,3,4,5$, and $\mu=1, \ldots, 6$

\section{Specific Heat}

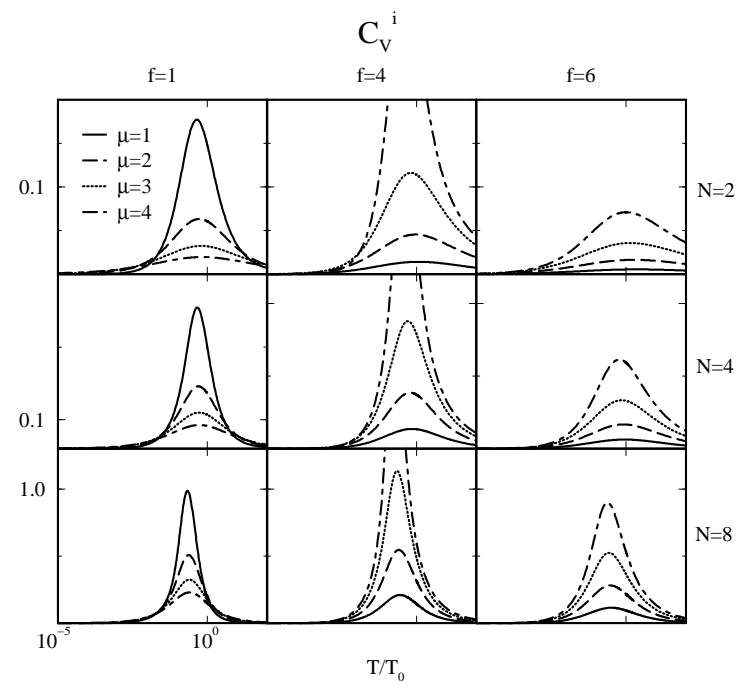

FIG. 8. Impurity contribution to the specific heat, $C_{V}^{i}$, for different values of $N, f$, and $\mu$
Next, we compute the contribution to the specific heat. Results for different values of the parameters are shown in Fig. 8. The largest maximum of $C_{V}^{i}$ corresponds to $\mu=f$. Also, the size of the curve grows with $N$.

We have also evaluated the subleading contribution to the linear coefficient of the specific heat, $\gamma^{i}=C_{V}^{i} / T$ for $f=2 \mu=2, N>f$, and we have plotted it in Fig. 9. The points fit power-law curves with exponents $(N-$ $f) /(N+f)$, derived previously (see Eqs. (3.29), (3.30), and $(3.32))$. . This is another clear indication that for $N>f$, the overscreened cases are not Fermi liquid fixed points.

$\mathrm{f}=2$

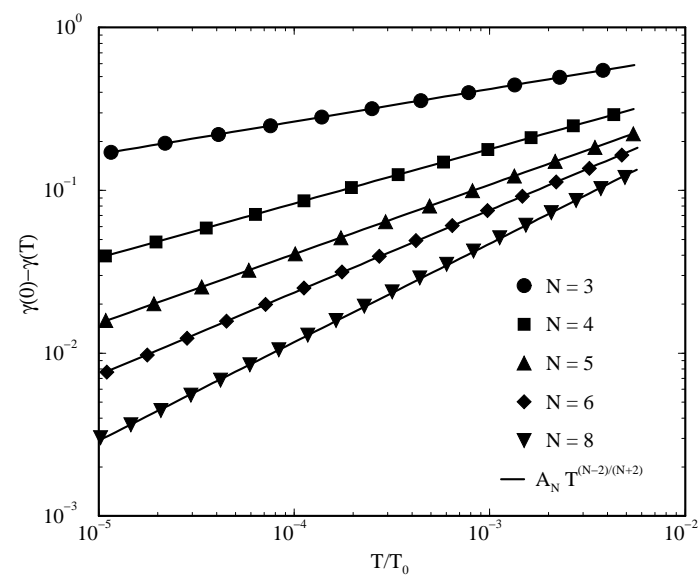

FIG. 9. Subleading contribution to $\gamma(T)=C_{V}^{i} / T$, for $f=2, \mu=1$, and $N>f$ vs. $T$, in a Log-Log graph. The symbols correspond to the numerical calculation. The lines correspond to power-law fits with exponents $(N-2) /(N+2)$.

\section{Magnetic Susceptibility}

Next, we have studied $T \chi^{i}$ for different values of the parameters, and plotted the results on Fig. 10. As with the entropy, the qualitative behavior depends only on the values of $\mu$ and $f$. The difference in behavior between underscreened and overscreened cases becomes more clear here: whereas the magnetic moment is partially quenched in the former case, the overscreened case is characterized by a totally quenched moment, even though there is a non-zero residual entropy. This can be seen in Fig. 10 for the curves with $f=1$ and $f=4$.

The magnetic susceptibility is plotted in Fig. 11. The curves with $f=\mu$ have a constant $\chi^{i}$ at low-T and for $N>2$ they have a maxima near $T \sim T_{0}$. This is a special feature of the completely screened case. We see that in the overscreened case with $N>f$, the susceptibility tends to a finite value as $T \rightarrow 0$, while it diverges when $N \leq f$. When $N=f$, the divergence is logarithmic, whereas it is power-law for $N<f$, with an 
exponent $\beta>-1$. The largest divergence corresponds to the underscreened case with $1 / T$ behavior. All these results coincide with those of the previous analytic study, Eqs. (3.29), 3.30), and (3.32).

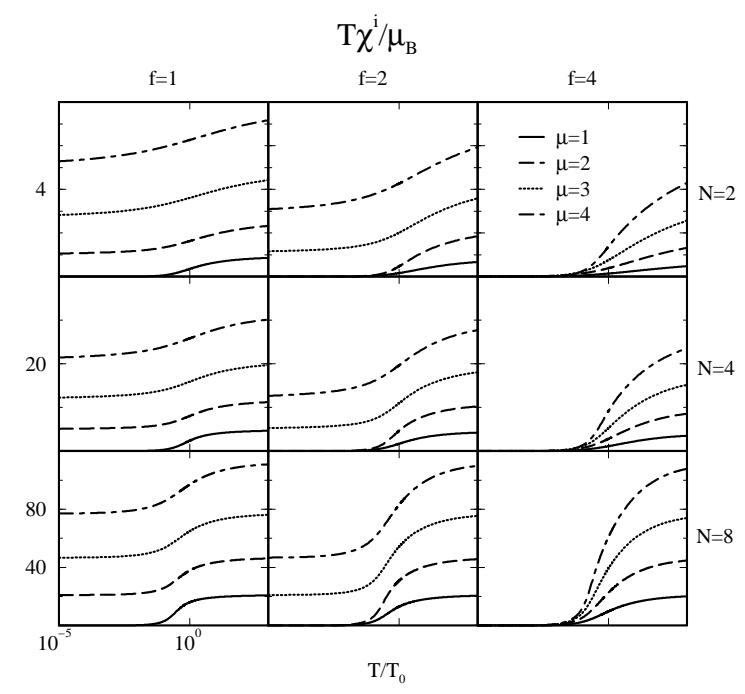

FIG. 10. $T \chi^{i}$ vs. T, for different values of $f, N$, and $\mu$

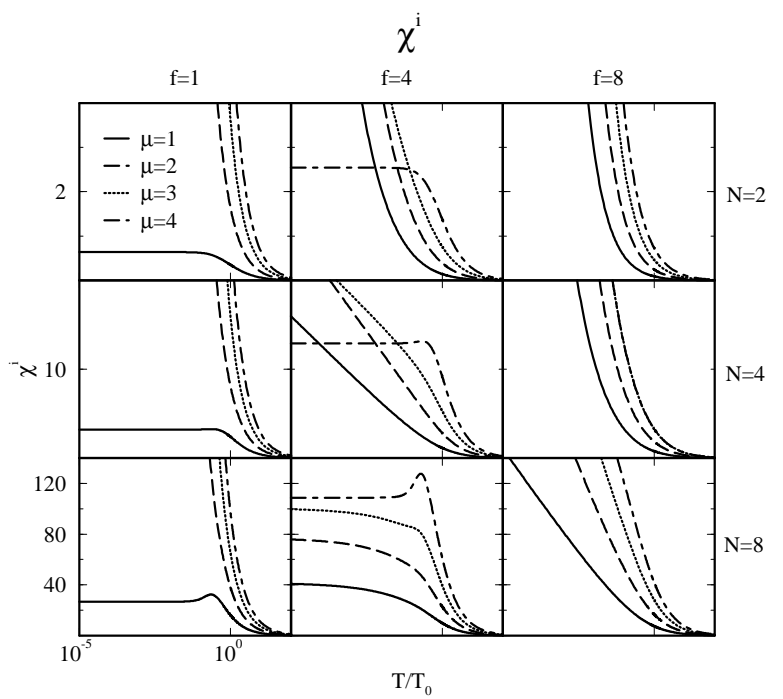

FIG. 11. Impurity contribution to the magnetic susceptibility, $\chi^{i}$ vs. $T$, for different values of $f, N$, and $\mu$.

In Fig. 12 we show $\chi^{i}$ for different values of $N$ for the cases $\mu=f=1$ and $\mu=f=4$. We have rescaled the curves dividing by $\chi^{i}(0)$. It is quite apparent that the behavior is the same in both cases.

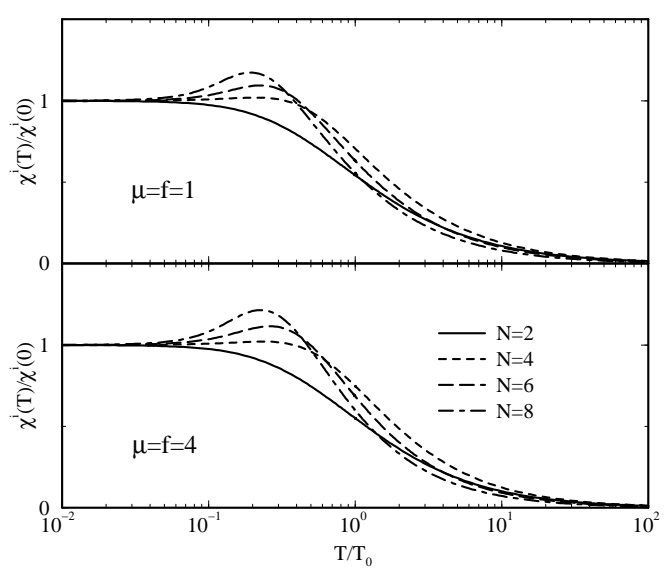

FIG. 12. $\chi^{i}(T) / \chi^{i}(0)$ vs. $T$ for different values of $N$, in two completely screened cases, $\mu=f=1$, and $\mu=f=4$.

$\mathrm{N}=4, \mu=1$

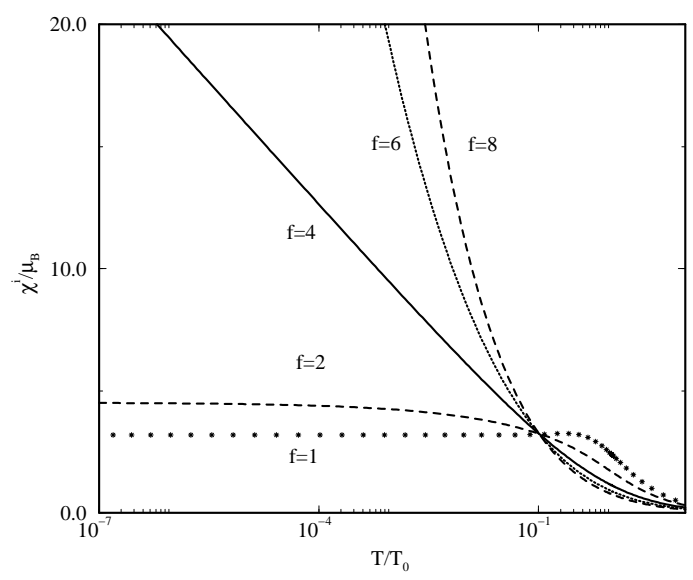

FIG. 13. $\chi^{i}$ vs. $T$ for $N=4, \mu=1$, and $f=1,2,4,6,8$.

In Fig. 14 we consider $N=8, f=7$ and $\mu=1, \ldots, 7$. Even though $\chi^{i}(0)$ is finite, it is clear that the behavior of the overscreened case is quite different from that of the completely screened case, and that the subleading terms have an important contribution below the crossover temperature.

We can also see the power-law behavior of the subleading term of $\chi^{i}$ in Fig. 15. The values for the exponents agree with the values obtained analytically, i.e. $(N-f) /(N+f)$. 
$\mathrm{N}=8, \mathrm{f}=7$

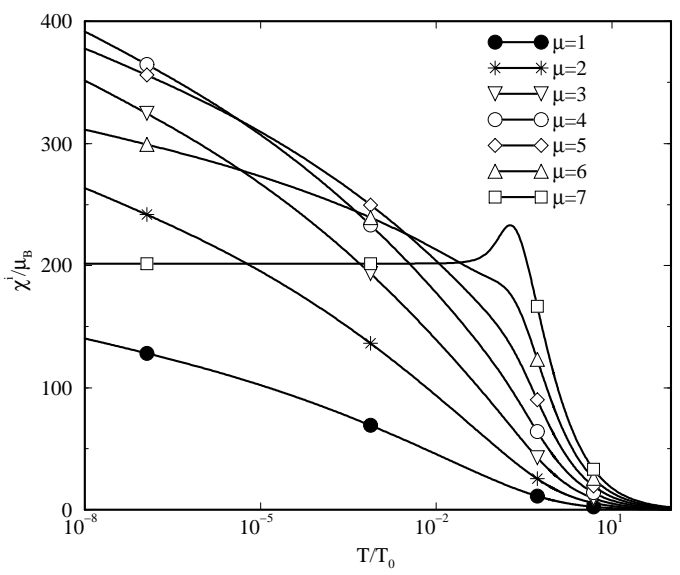

FIG. 14. $\chi^{i}$ vs. $T$ for $N=8, f=7$, and $\mu=1, \ldots 7$.

$\mu=1$

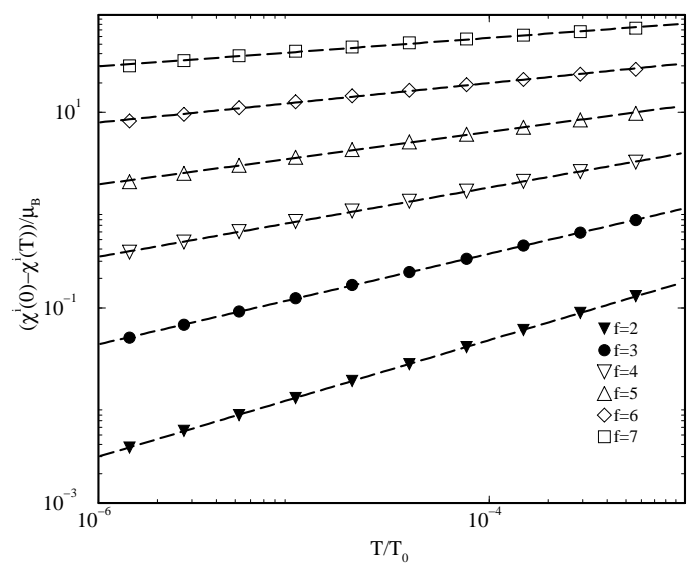

FIG. 15. Subleading $\mathrm{T}$ dependence of $\chi^{i}$ for $N=8$, $f=2, \ldots, 7, \mu=1$. The lines correspond to power law fits.

\section{Wilson Ratio}

We have calculated the Wilson ratio, defined as

$$
R \equiv \frac{\pi^{2} k_{B}^{2}}{\left(N^{2}-1\right) \mu_{B}^{2}} \frac{T \chi^{i}}{C_{V}^{i}}
$$

The quantity $R$ has a well-defined meaning only for $T=$ 0 . However, in Fig. 16 we have plotted the quantity $R(T)$, to show the difference between the $N<f$ and the $N>f$ sectors. In the former case, $(N=2, f>$ 1 ), the value for the overscreened case is much larger than the value of the completely screened case (notice the difference in vertical scales), whereas in the latter case $(N=8)$, the curves converge to the completely screened value. Notice that for $N=4$ there is a change in behavior as we go from $f<N$ to $f>N$.
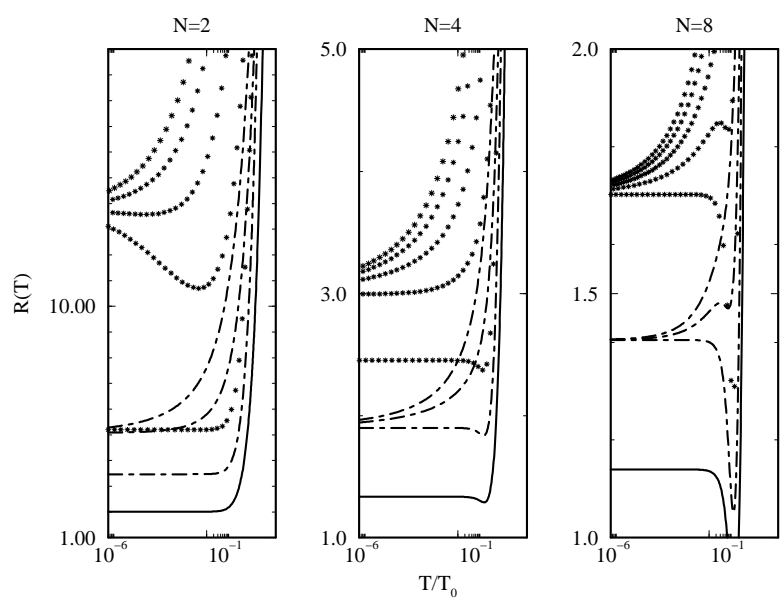

FIG. 16. $R(T)$ as a function of temperature for $N=2,4,8$, $\mu=1, \ldots, f$ and $f=1,3,5$.

Next we plot the values of $R$ for completely screened cases, $\mu=f$ (Fig. 17). We see that the values obtained fit the function

$$
R=\frac{N(N+f)}{N^{2}-1} .
$$

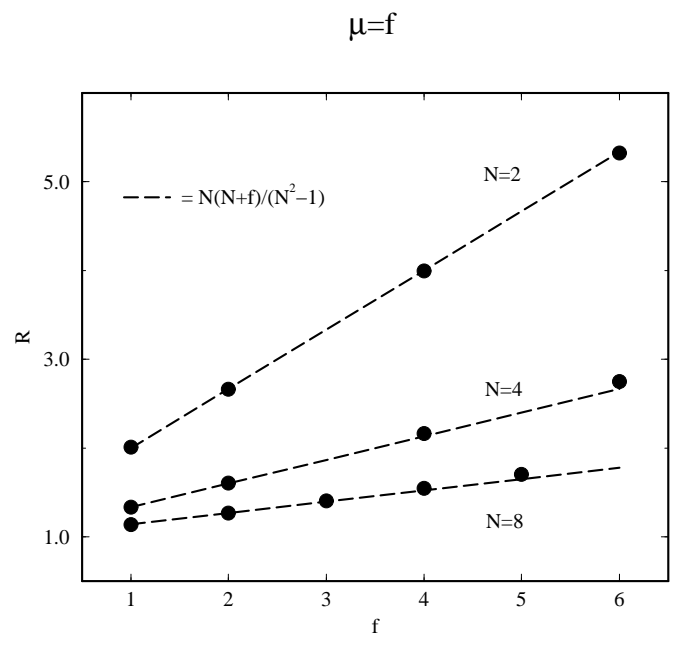

FIG. 17. Wilson Ratio $\mathrm{R}$ for $\mu=f$. The points have been obtained from the numerical solution. The lines correspond to fits with the function $N(N+f) /\left(N^{2}-1\right)$.

We have also obtained values of $R$ for the overscreened 
case $(\mu<f)$, in Fig. 18. There are clear differences between the $f<N$ and the $f>N$ cases, as we have already pointed out. For $f<N$, the value of $R$ coincides with the value for $\mu=f$, and agree with (4.2). The dominant contribution to $R$ comes from the constant terms in $\chi^{i}$ and $C_{V}^{i} / T$. For $f>N, R$ contains mainly the coefficients of the divergent parts, and have a different functional behavior.

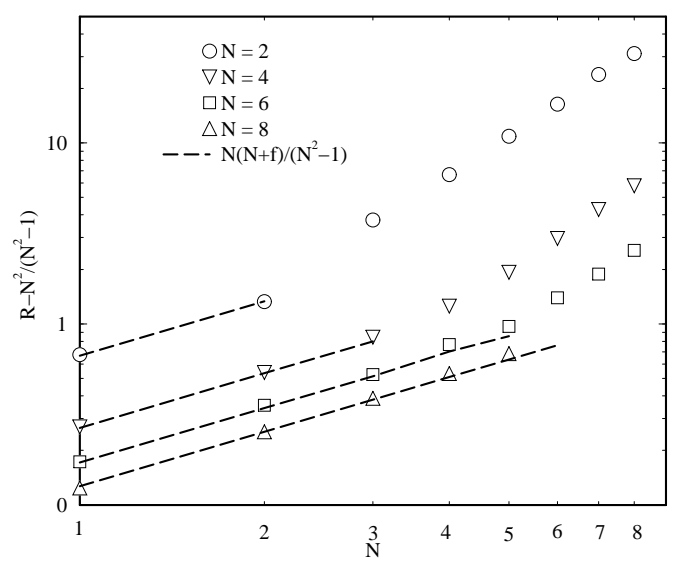

FIG. 18. Wilson Ratio $R$ for $\mu<f$. See the caption in the previous figure.

\section{Channel Anisotropy}

We end the discussion of the numerical results by showing an example of channel anisotropy. We have taken the case $N=3$, with original flavor symmetry $S U(6)$, broken down to $S U(4) \times S U(2)$ (Fig. 19) Two scales appear in this problem: $T_{6}$, and $T_{4}$. Accordingly, the TBA equations have driving terms at $n=4,6$.

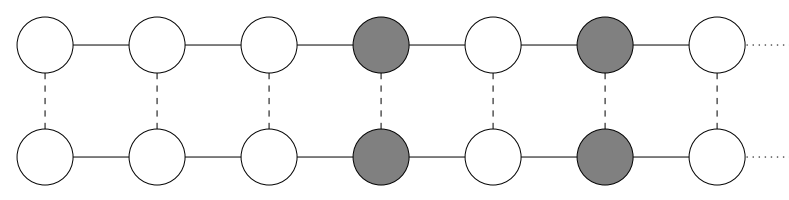

FIG. 19. Diagrammatic representation of the TBA equations for a system with $N=3$, and flavor symmetry $S U(6)$ broken down to $S U(4) \times S U(2)$. Two driving terms appear at $n=4,6$

We have chosen very small anisotropy, $T_{4} / T_{6}=10^{-6}$. In Fig. 20 we have plotted the entropy for different values of $\mu$. There are three different regions: When $T>>$ $T_{6}$, the impurity behaves like a free moment. Around $T \sim T_{6}$ there is a crossover to an overscreened region (when $\mu<6$ ), characterized by a $S U(6)$ flavor symmetry. The pairs of curves $(\mu, 6-\mu)$ merge. Notice also that $\mu=6$ is completely screened. There is a second crossover around $T \sim T_{4}$ to a region characterized by $S U(4)$ flavor symmetry for $\mu<4$ and by $S U(2)$ flavor symmetry for $4<\mu<6$. Only the curves for $\mu=1$ and $\mu=4-1=3$ coincide now. Also, the value of the residual entropy for $\mu=1$, is that of an effective $\mu=1$ in a $S U(3) \times S U(2)$ model. The entropy for $\mu=4$ goes to 0 with $T$ since the system becomes screened for $T \ll T_{4}$.

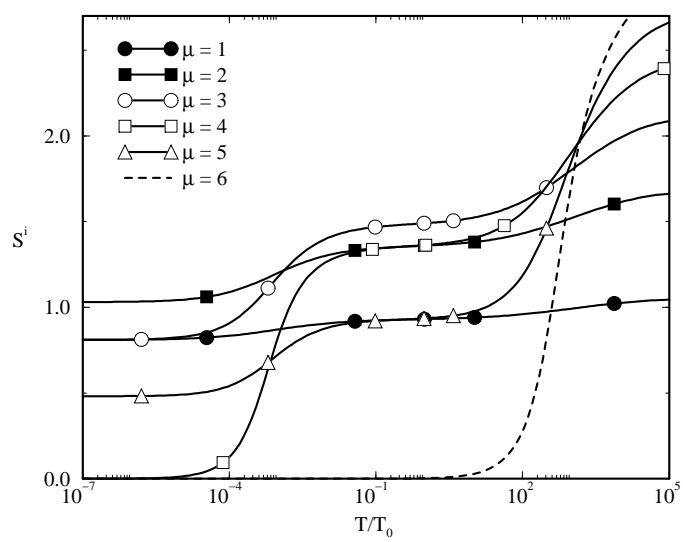

FIG. 20. Entropy vs. T, for $\mu=1, \ldots, 6$

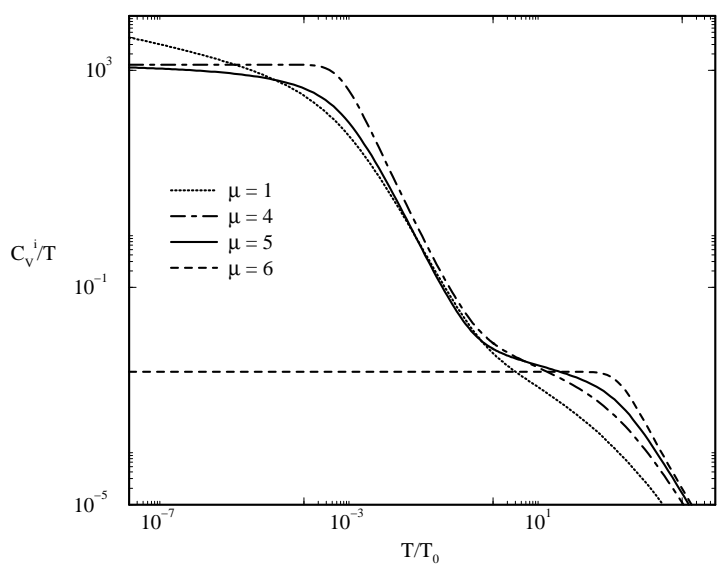

FIG. 21. $C_{V}^{i} / T$ vs. T, for $\mu=1,4,5,6$.

Finally, we have plotted $C_{V}^{i} / T$ for several $\mu$ in Fig. 21. When $T_{4}<<T<<T_{6}$, the behavior of the system is characterized by $f=6$ : increasing value of $C_{V}^{i} / T$ for $\mu<6$, constant behavior for the screened case, $\mu=6$. 
Near $T_{4}$ the curves for $\mu<6$ have a similar behavior as those for free moments (however, such behavior is not found in the curves for the magnetic susceptibility). We can see three different behaviors in the region $T \ll T_{4}$. For $\mu=1$ the curve diverges with a power law, as one would expect for $f_{\text {eff }}=4>N=3$. The curve for $\mu=4$ is flat, since the impurity is screened. Finally, for $\mu=5$, the curve increases slowly, converging to a constant value, as one would expect from an effective $f_{\text {eff }}=2<N=3$.

\section{PHYSICAL REALIZATIONS OF THE $S U(N) \times S U(F)$ MODEL}

In the present section we shall concentrate on the possible physical realizations and applications of the $S U(N) \times S U(f)$ Coqblin-Schrieffer model.

In Subsections. $\mathrm{VA}, \mathrm{VB}$, and $\mathrm{VC}$ we analyze the so called N-level system (NLS) model, a generalization of the two-level system model 2 by means of a systematic $1 / f$ expansion. In this context the flavor degeneracy is associated with the physical spin of the electron. First we establish a mapping of the NLS model to the multichannel Coqblin-Schrieffer model (MCCS model) by analyzing the low-energy fixed point of its scaling equations. While our procedure gives a systematic expansion only for the case $f>N$ we shall argue that the same mapping should apply for the cases $f \leq N$.

In the limit $f>N$ we are able to determine the full operator content of the fixed point. This enables us to calculate the scaling of the different physical quantities at low temperatures in Subsection $\mathrm{VQ}$. As we shall see there are some subtle differences between the two models and while most of the physical quantities show the same dependence, the scaling of the specific heat may be different. The origin of these differences will be discussed in detail.

Finally, based on the results of Subsections $\mathrm{VA} \mathrm{VG}$, we discuss in Subsection VD some physical systems providing possible candidates for the realization of the MCCS-model.

\section{A. The N-level system model and its low-energy fixed point}

The NLS model has been constructed as a generalization of the two-level system model2 24 to describe the tunneling of a heavy particle among $N$ not necessarily equivalent positions labeled by $a=\{1, . ., N\}$, and strongly coupled to the conduction electrons. At low temperatures the motion of the heavy particle can be described by the effective Hamiltonian

$$
H_{\mathrm{hp}}=\sum_{a, b=1}^{N} \chi_{a}^{+} \Delta^{a b} \chi_{b}
$$

where $\chi_{a}^{+}$creates a pseudofermion 25 corresponding to the heavy particle site $a$ and $\Delta^{a b}$ is the tunneling amplitude between positions $a$ and $b$. If no external stress is present then the diagonal part of $\Delta^{a b}$ vanishes: $\Delta^{a a}=0$ $(a=1, . ., N)$, when the $N$ positions are equivalent due to the symmetry of the NLS. The electronic part of the Hamiltonian and the coupling of the heavy particle to the conduction electrons take the general form:

$$
\begin{aligned}
H_{\mathrm{el}} & =\sum_{\epsilon n m} \epsilon c_{\epsilon n m}^{+} c_{\epsilon n m}, \\
H_{\mathrm{e}-\mathrm{hp}} & =\sum_{\substack{a, b, n, n^{\prime} \\
\epsilon, \epsilon^{\prime}, m}} c_{\epsilon n m}^{+} \chi_{a}^{+} V_{n n^{\prime}}^{a b} \chi_{b} c_{\epsilon^{\prime} n^{\prime} m}
\end{aligned}
$$

where the operators $c_{\epsilon n m}^{+}$create conduction electrons with energy $\epsilon$, orbital quantum number $(\sim$ angular momentum) $n=1,2, . ., \infty$, and spin $m$. For the sake of simplicity the electronic density of states $\varrho(\epsilon)$ is assumed to be constant $\varrho_{0}$, between the high- and low-energy cutoffs, $D$ and $-D$, independently of the flavor and orbital quantum numbers. While in the physical case only $m= \pm$ is possible corresponding to the two different spin directions, for technical reasons in the following we assume that the electron spin $m$ can take $f$ different values: $m=1, . ., f$.

This model has a structure similar to Eq. (2.1) but there are some important differences. The 'spin index' $a^{\prime}=1, \ldots, N$ of the impurity in Eq. (2.1) is now replaced by the 'site index' $a=1, . ., N$ of the heavy particle. Moreover, in the NLS case the orbital index $n$ (replacing the 'spin index' $a=1, \ldots, N$ of the conduction electrons in the $S U(N) \times S U(f)$ model) now ranges from one to infinity since the conduction electrons may have any orbital momentum. Furthermore, the couplings are highly anisotropic in orbital indices and no $S U(N)$ symmetry is present at this level. Finally, in the NLS model the scattering is diagonal in the real spin index $m$ which plays now the same role as the flavor in Eq. (2.1).

The diagonal couplings, $V_{n n^{\prime}}^{a a}$ describe simple potential scattering of the conduction electrons by the heavy particle sitting in position $a$. On the other hand, the offdiagonal matrix elements, $V_{n n^{\prime}}^{a b}$ with $a \neq b$ correspond to the so called "assisted tunneling" processes. Here the heavy particle is tunneling from one site to another while a conduction electron is scattered by it. The combination of these two processes leads ultimately to the generation of an orbital Kondo effect and a strongly correlated ground state. 2 :5

In the following we shall carry out a large $f$ analysis to determine the low-energy fixed point of the NLS model. While our procedure is strictly valid only in the case $f>$ $N$, in the end of the subsection we shall argue that our results are very general and they should apply even for the cases $f \leq N$.

To carry out a $1 / f$ analysis of the NLS model, as a next step, we construct the next to leading logarithmic scaling equations using a generalized multiplicative renormaliza- 
tion group technique. 0 As discussed in Refs. 1, 17, 18, 26, the leading logarithmic equations give the leading term in a systematic $1 / f$ expansion and become exact in the $f \rightarrow$ $\infty$ limit. In the multiplicative renormalization group method one exploits the existence of a non-trivial transformation in the space of the Hamiltonians, $D \rightarrow D^{\prime}$, $V_{n n^{\prime}}^{a b} \rightarrow V_{n n^{\prime}}^{a b}$, and $\Delta^{a b} \rightarrow \Delta^{a b}$ that leaves the pseudofermion Green's function $\mathcal{G}^{a b}$ and the pseudofermionconduction electron vertex function $\Gamma_{n n^{\prime}}^{a b}(\omega)$ invariant:

$$
\begin{aligned}
\mathcal{G}\left(\omega, V^{\prime}, \Delta^{\prime}, D^{\prime}\right) & =A \mathcal{G}(\omega, V, \Delta, D) A^{+}, \\
\Gamma\left(\omega, V^{\prime}, \Delta^{\prime}, D^{\prime}\right) & =\left[A^{+}\right]^{-1} \Gamma(\omega, V, \Delta, D) A^{-1} .
\end{aligned}
$$

In these equations $A$ denotes an $N \times N$ matrix independent of the energy variables, $\omega$ and $T$, and acting in the site indices: $A=A^{a b}\left(V^{\prime}, \Delta^{\prime}, D^{\prime} / D\right)$. By means of the transformation Eq. (5.3) one can generate effective Hamiltonians that describe the system's behavior below the energy scale $D^{\prime}$. The generated effective Hamiltonians usually (in a renormalizable theory) turn out to be much simpler than the original one.

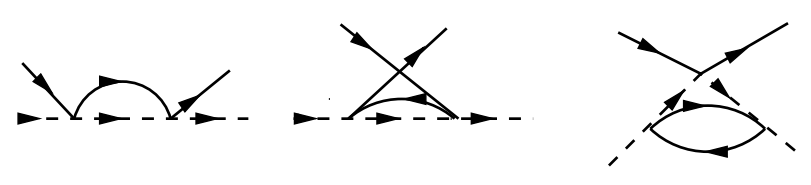

a.

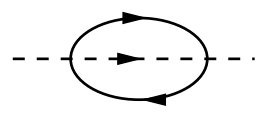

b.

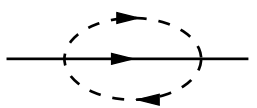

C.
FIG. 22. The leading logarithmic vertex and self-energy diagrams generating the next to leading logarithmic scaling equations. Continuous and dashed lines represent the conduction electron and pseudofermion Green's functions.

To make use of the invariance property Eq. (5.3) one first has to construct the lowest grder vertex and pseudofermion self-energy correctiond arising from the diagrams in Fig. 22. a and b,

$$
\begin{aligned}
\Sigma^{a b} & =-f \ln \frac{D}{\omega}\left(\delta^{a b} \omega \operatorname{tr}\left\{\underline{v}^{c d} \underline{v}^{d c}\right\}-\operatorname{tr}\left\{\underline{v}^{a c} \Delta^{c d} \underline{v}^{d b}\right\}\right) \\
\varrho_{0} \underline{\Gamma}^{a b} & =\underline{v}^{a b}-\ln \frac{D}{\omega}\left(\left[\underline{v}^{a c}, \underline{v}^{c b}\right]-f \operatorname{tr}\left\{\underline{v}^{a c} \underline{v}^{d b}\right\} \underline{v}^{c d}\right),
\end{aligned}
$$

where $\varrho_{0}$ is the density of states at the Fermi level, and a matrix notation has been introduced for the dimensionless couplings $\varrho_{0} V_{n n^{\prime}}^{a b} \rightarrow \underline{v}^{a b}$. The symbol [ , ] stands for the commutator, the trace operator $\operatorname{tr}\{\ldots\}$ is acting in the electronic indices, and a summation must be carried out over repeated indices. Then, substituting Eq. (5.4) into Eq. (5.3) and reducing the bandwidth $D$ by an infinitesimal amount one can deduce the infinitesimal renormalization group transformations for the couplings:

$$
\begin{aligned}
\frac{d \Delta^{a b}}{d x}= & -\frac{1}{2} f\left[\operatorname{tr}\left\{\underline{v}^{a c} \underline{v}^{c d}\right\} \Delta^{d b}+\Delta^{a c} \operatorname{tr}\left\{\underline{v}^{c d} \underline{v}^{d b}\right\}\right. \\
& \left.-2 \operatorname{tr}\left\{\underline{v}^{a c} \Delta^{c d} \underline{v}^{d b}\right\}\right] \\
\frac{d \underline{v}^{a b}}{d x}= & -\left[\underline{v}^{a c}, \underline{v}^{c b}\right]+\frac{1}{2} f\left(2 \operatorname{tr}\left\{\underline{v}^{a c} \underline{v}^{d b}\right\} \underline{v}^{c d}\right. \\
& \left.-\operatorname{tr}\left\{\underline{v}^{a c} \underline{v}^{c d}\right\} \underline{v}^{d b}-\underline{v}^{a c} \operatorname{tr}\left\{\underline{v}^{c d} \underline{v}^{d b}\right\}\right),
\end{aligned}
$$

where the dimensionless scaling variable $x=\ln \left(D_{0} / D\right)$ has been introduced, $D_{0}$ being the initial (real) bandwidth cutoff of the model.

These scaling equations have to be solved with the boundary condition that the couplings are equal to their bare values at $x=0$, and they loose their validity if the reduced bandwidth $D$ becomes smaller than any smallenergy scale present, $T, \omega, \Delta$. Note that, up to the next to leading logarithmic order, the splittings $\Delta^{a b}$ do not occur in Eq. (5.6) explicitly, and they provide only a low-energy cutoff for the scaling. To be explicit, there is an energy scale, $D^{*}=T^{*}$ that we call the freezing temperature, where the renormalized splitting becomes of the same order of magnitude as the reduced bandwidth: $\Delta^{a b}\left(D^{*}\right) \sim D^{*}$. Below this energy scale the orbital motion of the NLS is usually frozen out (see the discussion in the end of this Section), and the couplings may be replaced by their values at $T^{*}$.

For the moment let us forget about Eq. (5.5) and concentrate on the scaling of the $\underline{v}^{a b}$ 's, Eq. (5.6). This equation cannot be solved generally, but one can convince oneself very easily that if the 'assisted tunneling' matrix elements $\underline{v}^{a b}(a \neq b)$ do not vanish then the electron-NLS complings start to increase and lead to a Kondo effect 2.8 The scaling of the norm of the couplings, $\sum_{a, b}\left\|\underline{v}^{a b}\right\|$ is shown in Fig. 23 for a symmetrical six-state system, where the coupling constants have been estimated using similar methods as in Ref. 2. As one can see, a Kondo effect occurs around the Kondo scale $T_{K} \sim D_{0} e^{-x_{c}} \sim 10 K$, where $x_{c}=\ln \left(D_{0} / T_{K}\right)$ denotes the value of the scaling parameter at which the crossover from weak to strong coupling occurs. Our numerical investigations for various model parameters and different values of $N$ show that the structure of the stable lowtemperature fixed point the couplings scale to is independent of the initial couplings and only depends on the value of $N$ as long as no special symmetry is assumed for the $\underline{v}^{a b}$ 's.

In what follows we shall show that this stable lowenergy fixed point of Eq. (5.6) has the structure of the defining representation of the $S U(N)$ Lie algebra. To be precise we first observe that the operators $\mathcal{O}^{a} \sim$ $\delta^{a b} \sum_{c} \underline{v}^{c c}$ are invariant under scaling. Therefore the $\underline{v}^{a b}$ 's can be divided into two parts, $\underline{\tilde{v}}^{a b}$ and $\underline{M}^{a b}$ where $\sum_{a} \underline{\tilde{v}}^{a a}=0$ and $\underline{M}^{a b}$ is built up from the previously mentioned constants of motions, $\mathcal{O}^{a}$. Then as we shall see, 
at the stable fixed points of Eq. (5.6) the $\underline{\tilde{v}}^{a b}$ 's can be written as

$$
\left(\underline{\tilde{v}}^{a b}\right)_{\mathrm{fp}}=\frac{1}{f}\left(\begin{array}{cc}
\underline{L}^{a b} & 0 \\
0 & 0
\end{array}\right),
$$

where the $\underline{L}^{a b}$ 's satisfy the standard $S U(N)$ Lie algebra,

$$
\left[\underline{L}^{a b}, \underline{L}^{c d}\right]=\delta^{a d} \underline{L}^{c b}-\delta^{c b} \underline{L}^{a d} .
$$

and are unitary equivalent to the defining representation: $L_{n n^{\prime}}^{a b} \sim \delta_{n^{\prime}}^{a} \delta_{n}^{b}-\frac{1}{f} \delta^{a b} \delta_{n n^{\prime}}$. This statement is also demonstrated in Fig. 23 where the scaling of the 'algebra coefficient' $\alpha=\sum_{a, b, c, d}\left\|f^{2}\left[\underline{\tilde{v}}^{a b}, \underline{\tilde{v}}^{c d}\right]-f \delta^{a d} \underline{\tilde{v}}^{c b}+f \delta^{c b} \underline{\tilde{v}}^{a d}\right\|$ is shown, measuring how well the fixed point algebra (5.8) is satisfied. As one can see in Fig. 23 for $D \ll T_{K}$ the algebra coefficient $\alpha$ vanishes and therefore, in an appropriate basis, the $\underline{\tilde{v}}^{a b}$ 's really simplify to the form in Eq. (5.7).

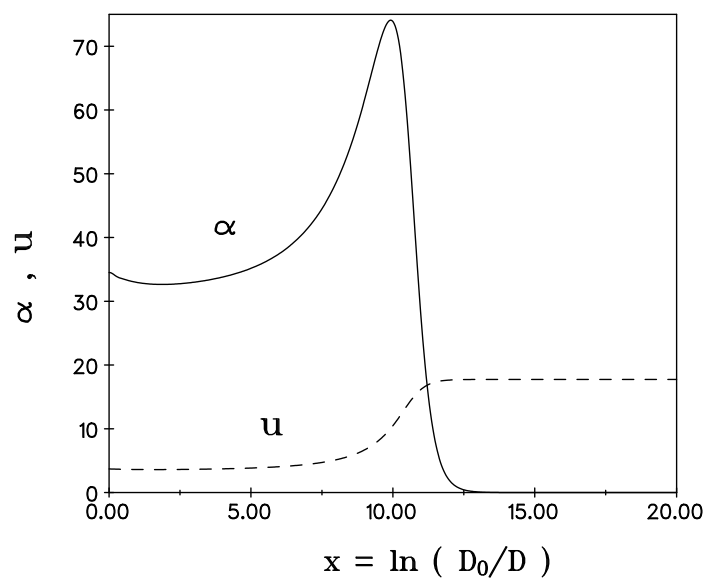

FIG. 23. Scaling of the norm of the dimensionless couplings, $u=\sum\left\|v^{a b}\right\|$ (dashed line), and of the algebra coefficient $\alpha$ (continuous line) for a 6 -state system with $f=2$.

Eq. (5.7) means that at the fixed point $\underline{\tilde{v}}^{a b}$ is given by Eq. (5.7) and apart from some potential scattering term the fixed point effective interaction can be written as

$$
H_{\mathrm{eff}}=V_{0} \sum_{\substack{a, b \\ \epsilon, \epsilon^{\prime}, m}} \chi_{a}^{+} c_{\epsilon b m}^{+} c_{\epsilon^{\prime} a m} \chi_{b},
$$

which is the same as the interaction term in Eq. (2.1). Note that while the initial model was very asymmetrical in the orbital space, at the fixed point only $N$ conduction electron angular momentum channels are coupled to the NLS and the fixed point effective Hamiltonian shows already an additional $S U(N)$ symmetry in the orbital sector as well. These statements are not true away from the fixed point where various kinds of irrelevant operators couple the NLS to the electrons and coupling to the other orbital channels is also relevant. The effective Hamiltonian is completely symmetrical in the NLS site index, which means that, e.g., the amplitude of assisted tunneling from site 1 to 6 in Fig. 23 is the same as the nearest neighbor assisted tunneling amplitude from site 1 to 2 , despite of their different geometrical position.

As it will become obvious in the next subsection, the analysis above is based on the possibility of a systematic $N / f$ expansion. Therefore, it is strictly valid in the $f>N$ case. However, one has several arguments that the effective Hamiltonian Eq. (5.9) is also adequate for the $N \leq f$ cases. First of all, in the case $N=2$ corresponding to the simpler case of multichannel Kondo model, it is well-known that for $f=1$ and $f=2$ the spin anisotropy of the couplings is irrelevant around the fixed point, 1,27 which has the same $S U(2)$ structure as Eq. (5.7). Furthermore, for $f=1$ but arbitrary $N$ one can easily prove following similar lines as Nozières and Blandin 1 that the isotropic fixed point, Eq. (5.9), is stable against spin (orbital) anisotropy. These observations together with our results for the $f>N$ case make it highly improbable that for $2 \leq f \leq N$ the NLS model would have a stable fixed point different from the one discussed above.

\section{B. Stability analysis of the $S U(N) \times S U(f)$ fixed point of the NLS model in the large $f$ limit}

The statement that $\underline{\tilde{v}}^{a b}$ is a fixed point of Eq. (5.6) is trivial. However, we also want to prove the stability of this fixed point analytically and find the irrelevant operators (determining the low-energy behavior of the model) around it. To this end we write the deviations from the fixed point in the form:

$$
\underline{\delta v}^{a b}=\left(\begin{array}{cc}
\underline{\varrho}^{a b} & \underline{t}^{a b} \\
\left(\underline{\underline{b}}^{a}\right)^{+} & \underline{\underline{\mu}}^{a b}
\end{array}\right),
$$

where the couplings $\underline{\varrho}^{a b}, \underline{t}^{a b}$, and $\underline{\mu}^{a b}$ are $N \times N, N \times \infty$, and $\infty \times \infty$ matrices, respectively. Substituting this expression into Eq. (5.6) one obtains the following linearized decoupled scaling equations:

$$
\begin{aligned}
\frac{d \underline{\mu}^{a b}}{d x} & =\frac{1}{f}\left(\delta^{a b} \underline{\mu}^{d d}-N \underline{\mu}^{a b}\right) \\
\frac{d \underline{\varrho}^{a b}}{d x} & =-\frac{1}{f}\left(\left[\underline{L}^{a d}, \underline{\varrho}^{d b}\right]+\left[\underline{\varrho}^{a d}, \underline{L}^{d b}\right]\right) \\
& +\frac{1}{2 f}\left\{2 \delta^{a b} \underline{\underline{\rho}}^{d d}+2 \underline{L}^{c d} \operatorname{tr}\left\{\underline{\varrho}^{a c} \underline{L}^{d b}+\underline{L}^{a c} \underline{\underline{ }}^{d b}\right\}\right. \\
& -2 N \underline{\varrho}^{a b}-\underline{L}^{a c} \operatorname{tr}\left\{\underline{\varrho}^{c d} \underline{L}^{d b}+\underline{L}^{c d} \underline{\varrho}^{d b}\right\} \\
& \left.-\operatorname{tr}\left\{\underline{\varrho}^{a c} \underline{L}^{c d}+\underline{L}^{a c} \underline{\varrho}^{c d}\right\} \underline{L}^{d b}\right\} \\
\frac{d t^{a b}}{d x} & =-\frac{1}{f}\left(\underline{L}^{a d} t^{d b}-\underline{L}^{d b} t^{a d}\right)+\frac{1}{f}\left(\delta^{a b} t^{d d}-N t^{a b}\right) .
\end{aligned}
$$

The solution of Eq. (5.11) is trivial, since the operator $\underline{\mu}^{a b}$ can be decomposed as $\underline{\mu}^{a b}=\left(\underline{\mu}^{a b}-\delta^{a b} \frac{1}{N} \underline{\mu}^{c c}\right)+\left(\delta^{a b} \frac{1}{N} \underline{\mu}^{c c}\right)$, 
where the first operator scales like $T^{\lambda} \sim e^{-x \lambda}$ with a dimension $\lambda=\lambda_{\mathrm{sl}}=N / f$, while the second is marginal with $\lambda=0$. The detailed analysis of the other two equations is much more complicated, but one can still find their exact solutions due to the simple structure of the $\underline{L}^{a b}$ 's Here we only briefly discuss the results of this analysis.

It turns out that Eqs. (5.12) and (5.13) have an infinite number of zero modes that can be divided into two distinct classes. The first type corresponds to potential scattering off the NLS and can be written as

$$
\underline{\delta v}_{\text {pot }}^{a b}=\delta^{a b} \underline{\delta v},
$$

where $\underline{\delta v}$ denotes an arbitrary $\infty \times \infty$ Hermitian matrix. The rest of the zero-modes can be identified with the generators $\underline{\delta v_{\text {gen }}^{a b}}$ of the unitary transformations of the $S U(N)$ Lie algebra, Eq. (5.8),

$$
\underline{\delta v}_{\mathrm{gen}}^{a b}=\left(\begin{array}{cc}
\underline{\varrho}_{\mathrm{gen}}^{a b} & \underline{t}_{\mathrm{gen}}^{a b} \\
\left(\underline{t}^{b a}\right)_{\mathrm{gen}}^{+} & 0
\end{array}\right) .
$$

More precisely, the generators $\underline{\varrho}_{\text {gen }}^{a b}$ and $\underline{t}_{\text {gen }}^{a b}$ can be shown to satisfy in first order the equations:

$$
\begin{aligned}
& \underline{L}^{a b} \underline{\underline{g}}_{\text {gen }}^{c d}-\underline{L}^{c d} \underline{\underline{g}}_{\text {gen }}^{a b}=\delta^{a d} \underline{\underline{t}}_{\text {gen }}^{c b}-\delta^{c b} \underline{t}_{\text {gen }}^{a d}, \\
& {\left[\underline{L}^{a b}, \underline{\rho}_{\text {gen }}^{c d}\right]+\left[\underline{L}^{c d}, \underline{\varrho}_{\text {gen }}^{a b}\right]=\delta^{a d} \underline{\varrho}_{\text {gen }}^{c b}-\delta^{c b} \underline{\varrho}_{\text {gen }}^{a d},}
\end{aligned}
$$

from which it follows that the operators $\underline{\tilde{L}}^{a b}:=\underline{L}^{a b}+$ $\underline{\delta v} \underline{v}_{\text {gen }}^{a b}$ satisfy the same Lie algebra as the original $\underline{L}^{a b}$, s:

$$
\left[\underline{\tilde{L}}^{a b}, \underline{\tilde{L}}^{c d}\right]=\delta^{a d} \underline{\tilde{L}}^{c b}-\delta^{c b} \underline{\tilde{L}}^{a d} .
$$

The $\underline{\varrho}_{\text {gen }}^{a b}$ 's turn out to be the generators of the unitary transformations in the $N$-dimensional electronic subspace where the Lie-algebra Eq. (5.8) is realized, while the $\underline{t}^{a b}$ 's correspond to the rotations of this $N$ dimensional subspace.

All the other eigenoperators around the fixed point can be shown to be irrelevant. Very surprisingly, at least in the large $f$ limit, the leading irrelevant operators are quite different from the leading irrelevant operator of the $S U(N) \times S U(f)$ model (5.9), both in their structure and in their scaling dimension. They are living in the sector $\underline{t}^{a b}$ and they can be written as

$$
\underline{\delta v}_{1}^{a b}=\left(\begin{array}{c}
0 \\
\left(\underline{C}^{b a}\right)^{+} \\
\underline{C}^{a b}
\end{array}\right),
$$

where the $C_{c m}^{a b}$ 's satisfy $\sum_{a} C_{c m}^{a a}=0$ and $\sum_{b}\left(C_{c m}^{a b}-\right.$ $\left.C_{a m}^{c b}\right)=0$ with $a, b, c=1, . ., N$ and $n=N+1, N+2, . ., \infty$. These operators have a dimension

$$
\lambda_{1}=\frac{N-1}{f}+\vartheta\left(\frac{N^{2}}{f^{2}}\right),
$$

We remark at this point that the operators (5.18) do not exist in the two-level-system model, which is therefore completely equivalent to the corresponding $S U(2) \times$
$S U(f)$ model.18 As we shall see, these operators do not give a contribution to physical quantities like the resistivity or the impurity susceptibility but they influence the thermodynamic behavior of the model. We stress at this point, that their existence is strictly proven in the $f \rightarrow \infty$ limit. They are very probably present even in the $N<f$ case but it is an open question if they survive in the $N \leq f$ limit.

The impurity resistivity will be shown to be dominated by the subleading operators

$$
\underline{\delta v}_{\mathrm{sl}}^{a b} \sim\left(\begin{array}{cc}
\underline{Q}^{a b} & 0 \\
0 & \underline{S}^{a b}
\end{array}\right),
$$

where the matrices $\underline{Q}^{a b}$ and $\underline{S}^{a b}$ satisfy $\underline{Q}^{a a}=\underline{S}^{a a}=0$ and $Q_{d c}^{a b}=Q_{d c}^{a b}$. These operators have a dimension

$$
\lambda_{\mathrm{sl}}=\frac{N}{f}+\vartheta\left(\frac{N^{2}}{f^{2}}\right) .
$$

and the operator $(5.9)$ considered in the $S U(N) \times S U(f)$ model is also one of them. Furthermore, one has other even more irrelevant operators in the $\underline{t}$ sector of $\underline{\delta v}$ with a dimension $\lambda_{\text {ssl }}=(N+1) / f$ which give a subleading contribution to the physical quantities calculated.

In the previous considerations we did not take into account the presence of the splitting $\Delta^{a b}$ of the NLS. As we discussed already, this splitting results in the appearance of another low-energy scale, $T^{*}$. Below this the NLS cannot jump freely between its $N$ different positions. Since usually the ground state of the $N L S$ is non-degenerate in most cases a Fermi liquid state develops. In other words, the non-Fermi liquid $S U(N) \times S U(f)$ fixed point is unstable with respect to the splitting that usually drives the system towards a Fermi liquic ground state.

It has been argued very recently 28 that in special cases, due to some dynamical Jahn-Teller effect, e.g., the hopping amplitude $\Delta^{a b}$ might pick up and additional Berry phase, which could then result in a degenerate ground state with degeneracy $N^{\prime}$. Then the effective Hamiltonian at very low temperatures would be, of course, an $S U\left(N^{\prime}\right) \times S U(f)$ exchange model, and in the region $T \ll T^{*}$ all our previous considerations hold with the replacement of $N$ by $N^{\prime}$. Unfortunately, this Berry phase scenario is not very probable and therefore we expect the non-Fermi liquid behavior can be only observed in the restricted temperature (energy) range $T^{*}<\max \{T, \omega\}<$ $T_{K}$, i.e., when the freezing temperature is small enough.

Therefore it is very important to determine the realistic values of the freezing temperature. We estimated the freezing temperature by solving the scaling equations (5.5) and (5.6) numerically for the same symmetrical 6-level system as in Fig. 23. In this case the diagonal matrix elements $\Delta^{a a}$ vanish by symmetry. As one can see from Fig. 24, for a realistic NLS the renormalization of the hoppings $\Delta^{a b}$ is huge, and the situation $T^{*}<\max \{T, \omega\}<T_{K}$ can be reached quite easily. We note at this point that in our Hamiltonian we 
also neglected the contribution of two-electron scattering around the fixed point, which might be also releyant in the immediate neighborhood of the fixed point.28 However, these have a very small amplitude and they are scaled downwards in the first part of the scaling, $D>T_{K}$. Therefore, most likely their effect can be neglected compared to that of the splitting $\Delta^{a b}$, which provides the dominant mechanism driving the system to a Fermi liquid state.29

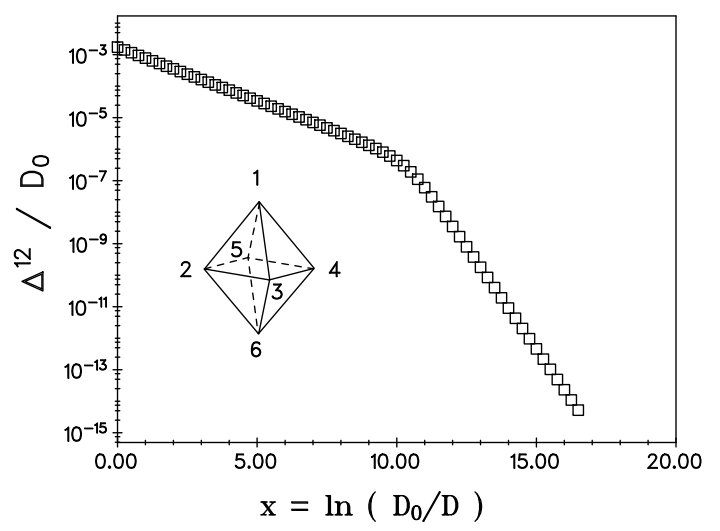

FIG. 24. Scaling of the dimensionless hopping amplitude, $\Delta^{12} / D_{0}$ for the same 6-state system as in Fig. 23. Inset: Numbering of the sites of the 6 -state system.

\section{Scaling of the physical quantities of the NLS model in the large $f$ limit}

Now we turn to the calculation of the physical quantities. In this Subsection we shall determine different thermodynamic quantities and the conduction electrons' scattering rate, $1 / \tau$, which is directly proportional to the impurity contribution to the electrical resistivity, $R_{\text {imp }}(T)$.

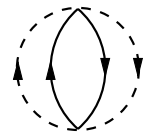

a.

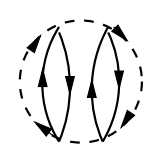

d.

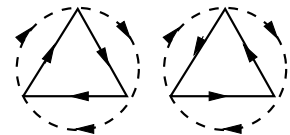

b.

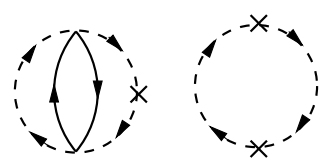

e.

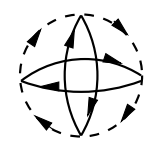

c.
FIG. 25. Diagrams generating the $1 / f^{2}$ corrections to the free energy. The crosses denote the counterterm.

To calculate a general physical quantity one should also calculate the renormalization coefficient $A$ in Eq. (5.3), a non-trivial task away from the fixed point. However, one can easily convince oneself that the factors $A$ and $A^{-1}$ in the free energy corrections in Fig. 25 and the electronic self-energy corrections in Fig. 22.c cancel exactly, and therefore these are scale invariant, and can be calculated by considering only the scaling equations (5.5) and (5.6).

For the sake of simplicity let us assume first that the highest low-energy scale is given by the temperature. To calculate a physical quantity at a temperature $T$ we apply a renormalization group transformation (5.3) with $D=D_{0}$ and $D^{\prime}=T$. Then in the new Hamiltonian all the logarithmic terms vanish since $\ln \left(D^{\prime} / T\right)=0$, and the different physical quantities are exclusively given by the non-logarithmic contributions of the corresponding diagrams. For a scale invariant quantity like the free energy, e.g., this implies that

$$
\begin{aligned}
& F_{\text {imp }}\left(D_{0}, T, \underline{v}_{0}^{a b}, \Delta_{0}^{a b}\right)= \\
& F_{\text {imp }}\left(T, T, \underline{v}^{a b}\left(\ln \frac{D_{0}}{T}\right), \Delta^{a b}\left(\ln \frac{D_{0}}{T}\right)\right),
\end{aligned}
$$

where on the right-hand-side no logarithmic corrections appear, but the renormalized couplings have to be used.

Therefore, in order to calculate the scaling behavior of the thermodynamic quantities our task is to determine the non-logarithmic parts of the different free energy diagrams. Since the fixed point couplings $\underline{v}_{\mathrm{fp}}^{a b}$ are proportional to $1 / f$ up to $1 / f^{2}$ order only the diagrams in Figs. 25.a-d. contribute. However, these diagrams contain divergent contributions originating from the finite part of the self-energy diagram in Fig. 22. b. These spurious divergences can be handled by a standard renormalization procedure, 30 by adding the following counterterm to the Hamiltonian:

$$
H_{\text {count }}=f 2 D \ln 2 \chi_{a}^{+} \chi_{c} \operatorname{tr}\left\{\underline{v}^{a b} \underline{v}^{b c}\right\} .
$$

This counterterm can also be interpreted as a renormalization of the bare parameters of the model, which should be used in Eq. (5.3) as the initial conditions. Then the counterterm contributions in Fig. 25.e cancel all the spurious divergences, and after a tedious calculation one obtains for the non-logarithmic part of the free energy:

$$
\begin{aligned}
F_{\mathrm{imp}} & =-T\left[\ln N+\frac{2 \pi^{2} f}{3 N}\left(\operatorname{tr}\left\{\underline{v}^{a b} \underline{v}^{b c} \underline{v}^{c a}-\underline{v}^{c a} \underline{v}^{b c} \underline{v}^{a b}\right\}\right)\right. \\
& -\frac{f^{2} \pi^{2}}{2 N}\left(\operatorname{tr}\left\{\underline{v}^{a b} \underline{v}^{c d}\right\} \operatorname{tr}\left\{\underline{v}^{b c} \underline{v}^{d a}\right\}\right. \\
& \left.\left.-\operatorname{tr}\left\{\underline{v}^{a b} \underline{v}^{b c}\right\} \operatorname{tr}\left\{\underline{v}^{c d} \underline{v}^{d a}\right\}\right)+\ldots\right] .
\end{aligned}
$$

Note that diagram (a) in Fig. 25 is proportional to $T^{2} / D$, and it does not give a contribution in the scaling limit.

Substituting the fixed point couplings Eq. (5.7) into Eq. (5.24) the fixed point entropy can be calculated as

$$
S_{\mathrm{imp}}=\frac{\partial F_{\mathrm{imp}}}{\partial T} \approx \ln N-\frac{N^{2}-1}{f^{2}} \frac{\pi^{2}}{6}
$$

which is just the expanded version of Eq. (3.18). Note that Eq. 5.25) gives the NLS contribution to the entropy 
only in the region $T^{*} \ll T \ll T_{K}$. Below $T^{*}$ the motion of the NLS is usually frozen out and the impurity entropy tends to zero, as expected for a Fermi liquid state.

The scaling of the free energy in the region $T^{*} \ll T \ll$ $T_{K}$. can be determined by expanding the $\underline{v}^{a b}$ 's around their fixed point values like Eq. (5.10) and substituting them into Eq. (5.24). It turns out that similarly to the multichannel Kondo and the two-level system case 17,18 only the second order terms in $\underline{\delta v}^{a b}$ contribute, and therefore in the temperature range $T^{*} \ll T \ll T_{K}$ in leading order the free energy and the specific heat scale as

$$
\begin{aligned}
& F_{\mathrm{imp}} \sim T\left(\frac{T}{T_{K}}\right)^{2 \lambda_{1}} \sim T^{\frac{2(N-1)}{f}+1}, \\
& c_{\mathrm{imp}} \sim\left(\frac{T}{T_{K}}\right)^{\frac{2(N-1)}{f}} .
\end{aligned}
$$

Below $T^{*}$ the Free energy generally shows a Fermi liquid behavior. This scaling behavior does not agree with the one obtained in the BA solution of the exchange model. However, we have to remark at this point, that according to our estimations the amplitude of the subleading operators in $\underline{\delta v}^{a b}$ is larger than that of the leading irrelevant operators. Therefore, one expects that there is a substantial energy region where the subleading operators dominate, and eventually it is also possible that they dominate the scaling of the free energy in the whole region $T^{*} \ll T \ll T_{K}$. Then the exponent $\lambda_{1}$ in Eq. (5.26) should be replaced by $\lambda_{\mathrm{sl}}$ and one obtains a scaling $c_{\text {imp }} \sim T^{2 N / f}$ which is in $1 / f$ order completely identical with the Bethe ansatz and conformal field theory results for the $S U(N) \times S U(f)$ model.

One can also easily determine the scaling of the splitting susceptibility $\chi_{\Delta}=\partial^{2} F_{\text {imp }} / \partial \Delta^{2}$ at $T=0$ for small $\Delta$ 's, where now $\Delta$ denotes the characteristic value of the splittings $\Delta^{a b}$. Investigation of the free energy diagrams Fig. 25 shows that the 'splitting magnetization' $M_{\Delta}=\partial F_{\mathrm{imp}} / \partial \Delta$ should be of the form:

$$
M_{\Delta}=m\left(\frac{\Delta}{D}, \underline{v}^{a b}\right) .
$$

The important point is that $\Delta$ is not scale invariant, but it rather behaves as

$$
\Delta^{\prime}=Z_{\Delta}\left(\frac{D_{0}}{D}, \underline{v}^{a b}\right) \Delta,
$$

where the factor $Z_{\Delta}$ should be determined by integrating Eq. (5.5). As a consequence, $M_{\Delta}$ is not scale invariant either, and it has to be rescaled under the RG transformation by the factor $Z_{\Delta}$. Therefore, applying the renormalization group transformation to Eq. 5.28) with $D^{\prime}=\Delta^{*}=T^{*}$ we obtain:

$$
M_{\Delta}=Z_{\Delta}\left(\frac{D_{0}}{\Delta^{*}}, \underline{v}^{a b}\right) \times m\left(1, \underline{v}_{\mathrm{fp}}^{a b}\right)
$$

where we assumed that $\Delta^{*} \ll T_{K}$ and thus the scaled couplings $\underline{v}^{a b}\left(D^{\prime}\right)$ can be replaced by their fixed point values. Since $m\left(1, \underline{v}_{\mathrm{fp}}^{a b}\right)$ is just a constant, the scaling of $M_{\Delta}$ is the same as that of the factor $Z_{\Delta}\left(\frac{D_{0}}{\Delta^{*}}, \underline{v}^{a b}\right)$. For very small $\Delta$ 's the scaling of $Z_{\Delta}$ can be easily determined from the fixed point form of the scaling equation (5.5)

$$
\frac{d \Delta^{a b}}{d x}=-\frac{N}{f} \Delta^{a b}
$$

and one obtains in leading order in $1 / f$ :

$$
M_{\Delta} \sim Z_{\Delta}\left(\frac{D_{0}}{\Delta^{*}}\right) \sim\left(\frac{\Delta^{*}}{T_{K}}\right)^{N / f} \approx\left(\frac{\Delta}{T_{K}}\right)^{N / f},
$$

in agreement with (3.29) and the conformal field theory results. 31 In higher order in $1 / f$ one also has to take into account the renormalization of the splitting in Eq. (5.32), $\Delta^{*} \sim \Delta^{1 /\left(1-\lambda_{\mathrm{sl}}\right)}$ and one obtains with $\lambda_{\mathrm{sl}}=\frac{N}{N+f} \approx$ $\frac{N}{f}-\frac{N^{2}}{f^{2}}$

$$
M_{\Delta} \sim \Delta^{\frac{\lambda_{s 1}}{1-\lambda_{s 1}}} \sim \Delta^{N / f},
$$

which is the exact result 16 , 37

Finally, we discuss the scaling of the electronic scattering rate, which we determine from the imaginary part of the electronic self-energy in Fig. 22.c. By assuming a finite impurity concentration $n_{i}$ and averaging over the position of the impurities and the orientation of the incoming electrons we obtain for the average scattering rate:

$$
\left\langle\frac{1}{\tau}\right\rangle=2 \pi n_{i}\left(2 D_{0}\right) \frac{1}{N} \operatorname{tr}\left\{\underline{v}^{a b} \underline{v}^{b a}\right\} .
$$

Note, that the factor $D_{0}$ arises from the inverse density of states $\varrho_{0}^{-1}$ and is invariant under scaling. Substituting into this equation $\underline{v}^{a b}=\underline{v}_{\mathrm{fp}}^{a b}+\underline{\delta v}^{a b}$ we see immediately that the leading irrelevant operators do not give a contribution to the electronic scattering rate which is dominated by subleading operators and scales like

$$
\begin{aligned}
& \frac{1}{\tau} \sim T^{\lambda_{\mathrm{s} 1}} \sim T^{N / f} \quad(\omega=0), \\
& \frac{1}{\tau} \sim \omega^{N / f} \quad(T=0) .
\end{aligned}
$$

In higher orders this result should be replaced by $1 / \tau \sim$ $T^{N /(f+N)}$ and $1 / \tau \sim \omega^{N /(f+N)}$.

\section{Discussion of the possible physical realizations of the NLS model}

The simplest possible realization of the $S U(N) \times S U(f)$ model is given by substitutional impurities in metals. These impurities may form tunneling centers 32.33 which then interact with the conduction electrons' band. An example of such a system is given by $P b_{1-x} G e_{x} T e .33$ The alloy $P b T e$ is a narrow gap semiconductor, but usually because of some intrinsic impurities it becomes metallic 
at low temperatures. Since the $G e^{2+}$ ions are smaller than the $\mathrm{Pb}^{2+}$ ions and they are also attracted by their nearest neighbor $T e^{2-}$ ions, they form 8-state systems, and according to the our discussions in Section $\mathrm{VA}$ they would be good candidates for the $S U(8) \times S U(2)$ model.

However, while an unambiguous logarithmic anomaly has been observed in the resistivity of these materials, 33 no non-Fermi liquid behavior has been detected. There may be several reasons for that. According to the results of the BA calculations in the case $N>f$ the non-Fermiliquid corrections are subleading, and the physical quantities have in leading order a Fermi liquid-like behavior. The subleading low-temperature behavior of these alloys has never been analyzed and the original measurements do not seem to be accurate enough to extract such detailed information. We are also not aware of any measurements of other physical quantities like the specific heat in the interesting concentration domain. Furthermore, PbTe has very complicated properties: it has a soft phonon mode that drives the system trough a ferroelectric phase transition as a function of the Ge concentration, and strong spin-orbit scattering which most likely spoils the $S U(2)$ symmetry of the electron spins as well. Moreover, the measurements have been carried out at relatively large $G e$ concentrations, where the interaction of the NLS's can not be neglected any longer.

It seems that in order to observe the non-Fermi liquid scaling much more accurate measurements should be carried out at even lower temperatures and lower Ge concentrations for several physical quantities and lower Ge concentrations. One could also try to find a better candidate. Since in the case of PbTe the formation of the NLS's is induced by the ionic attractions, we think that experimentalists should search among multicomponent metals, where interstitials can occur in the material.

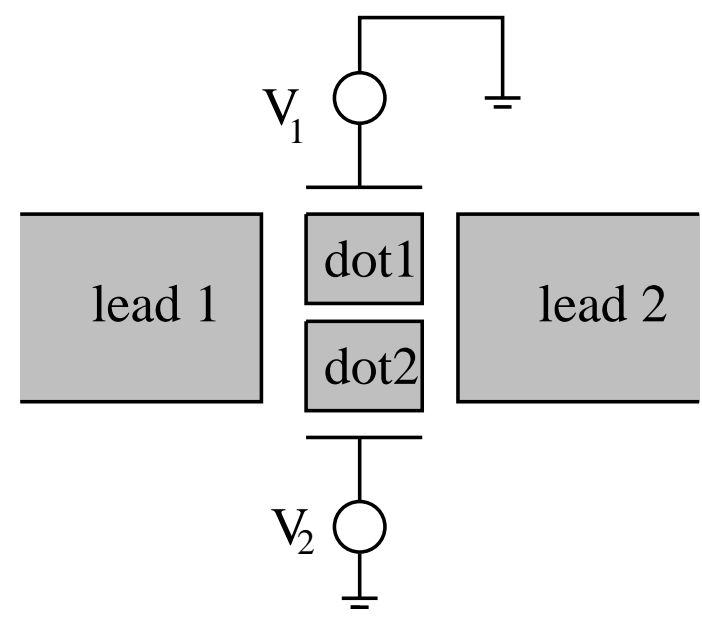

FIG. 26. A mesoscopic double dot system, candidate for the $S U(3) \times S U(2)$ model.

Similarly to the case of the two-channel Kondo model,34 another possible realization of the $S U(N) \times$
$S U(f)$ model could be possible by means of nanotechnologies. In Fig. 26 we show a double dot geometry which is a candidate for the realization of the $S U(3) \times S U(2)$ model. In the leads the electrons can be described as free particles:

$$
H_{\text {leads }}=\sum_{\alpha=l 1, l 2} \sum_{\epsilon \sigma} \epsilon c_{\epsilon \alpha \sigma}^{+} c_{\epsilon \alpha \sigma},
$$

where $\alpha=l 1, l 2$ refers to the two leads and $\sigma= \pm$ is the electron spin.

The Hamiltonian of the dots can be written as 35

$$
\begin{aligned}
H_{\mathrm{dots}} & =\sum_{\alpha=d 1, d 2} \sum_{\epsilon \sigma} \epsilon c_{\epsilon \alpha \sigma}^{+} c_{\epsilon \alpha \sigma} \\
& +\frac{\left(Q_{1}-V_{1} C_{1}\right)^{2}}{2 C_{\Sigma, 1}}+\frac{\left(Q_{2}-V_{2} C_{2}\right)^{2}}{2 C_{\Sigma, 2}}+\frac{Q_{1} Q_{2}}{C_{12}},
\end{aligned}
$$

where $\alpha=d 1, d 2$ is the index of the two dots, $Q_{1}$ and $Q_{2}$ are the charges of them, $V_{1}$ and $V_{2}$ denote the applied gate voltages in the Figure, and the $C$ 's denote different capacitances of the system.36 With a suitable choice of the gate voltages one can achieve that the ground state of the dots becomes three fold degenerate corresponding to the states $\beta:=\left(Q_{1}, Q_{2}\right)=(0,0),(0, e)$ and $(e, 0)$. Then the tunneling processes among the leads and the dots result in the simultaneous flips in the electrons orbital quantum number $\alpha=\{l 1, l 2, d 1, d 2\}$ and the charge variables $\beta$ which now take over the role of the orbital index of the NLS. Since the tunneling is diagonal in the electron's spin we have an additional $S U(2)$ degeneracy in the spin of the electrons. In sum, this system is a good candidate for the realization of the $S U(3) \times S U(2)$ model, where the $S U(3)$ fixed point symmetry corresponds to the three fold degenerate ground states of the dots.

\section{CONCLUSIONS}

We have studied the Multichannel Coqblin-Schrieffer model (MCCS) and its relation to the N-level system model (NLS). The properties of the MCCS model depend on both the spin and flavor symmetries, $S U(N)$ and $S U(f)$, as well as on the spin of the impurity.

We have performed both analytical and numerical studies of the model. As with the multichannel Kondo model, there are three different classes of fixed points depending on the spin of the impurity, $\mu$. The underscreened and completely screened fixed points $(\mu>f$ and $\mu=f$, respectively) have qualitative similar behavior to the analogous multichannel counterparts $(N=2)$.

There are overscreened fixed points. They display Non-Fermi liquid behavior: they have associated anomalous residual entropy and anomalous exponents in the low-temperature expansion of quantities like the specific heat and the magnetic susceptibility.

For an impurity with spin in the fundamental representation of $S U(N)$, the residual entropy, $\mathcal{S}^{i}$, is only a 
function of $N+f$ and $|\log (N / f)|$. Hence, there are different fixed points with the same value of $\mathcal{S}^{i}$. The exception corresponds to $N=f$, which yields the largest value of the residual entropy for fixed $N+f$.

The low-temperature thermodynamics are determined by the value of the ratio $\gamma=f / N$ alone, for any $\mu<f$. When $f \neq N$ we have

$$
\frac{C_{V}^{i}}{T}, \chi^{i} \propto \operatorname{ctn} .+\left(\frac{T}{T_{K}}\right)^{\frac{1-\gamma}{1+\gamma}}
$$

which diverge for $\gamma>1$, but remain finite for $\gamma<1$. When $N=f$, the power is replaced by a logarithm, as in the two-channel Kondo model. The constant terms in (6.1) are always present and are the dominant contributions in the completely screened case, $\mu=f$, and when $f<N$. The Wilson ratio in such cases is given by

$$
R=\frac{N(N+f)}{N^{2}-1}
$$

Channel anisotropy is a relevant perturbation. As the channel symmetry is reduced from $S U(f)$ to $S U\left(f^{\prime}\right)$, the entropy is quenched since $N+f$ decreases and $|\log (N / f)|$ increases. Likewise, a system with channel anisotropy might behave like a $f>N$ system at intermediate temperatures and flow at low- $T$ to a $f<N$ system.

Then we turned to the comparison of the MCCS model to the N-level system model (NLS), describing a heavy particle tunneling between $N$ different positions and interacting with the conduction electrons. We have shown that the low-energy fixed point of the NLS model is just the $S U(N) \times S U(f)$ MCCS model. Performing a $1 / f$ study of the NLS model we have analyzed the operator content of this low-energy fixed point, and the scaling properties of different physical quantities in the $N<f$ limit. We have shown in this limit that while the operator content of the NLS model is different from that of the MCCS model, apart from some subtle differences, the low-energy properties of the two models are the same. Especially, comparison with the exact results obtained in the first part of the paper and with the NCA calculations 16 show that the susceptibility, the residual entropy and the resistivity of the two models behave in the same way, and for reasonable physical parameters even the scaling of the specific heat is properly described by the MCCS model.

Finally, we discussed some possible physical realizations of the $S U(N) \times S U(f)$ models. First we discussed the case of tunneling interstitials in multicomponent metals such as $P b_{1-x} G e_{x} T e$ compounds. We pointed out that the low concentration of interstitials is essential to avoid strong inter-impurity interactions and keep the diagonal elements of the self-energy $\Delta^{a b}$ small. Secondly, we suggested a double quantum dot structure that could give an ideal realization for the $S U(3) \times S U(2)$ model.

\section{ACKNOWLEDGMENTS}

In the course of this investigation we learned of parallel work by A. Georges, O. Parcollet, G. Kotliar and A. Sengupta, using conformal field theory and large-N approach to study the same model. There is perfect agreement whenever comparison can be made. We are most grateful to the authors for many useful and enlightening discussions and sharing their results prior to circulation. Part of the work was carried out while N.A. was visiting the group of Physique Theorique at the ENS. It is a pleasure to thank the members of the group for their warm hospitality.

G.Z. would like to acknowledge useful discussions with D.L. Cox K. Vladár, A. Zawadowski, and A. Moustakas. He would like to thank the Magyary Zoltán Foundation and the Institut Laue-Langevin (Grenoble) for its hospitality, where part of the present work has been done.

A.J. would like to acknowledge useful discussions with R. Bulla, P. Coleman, F.H.L. Essler, A. Hewson, A.F. Ho, A. Lopez, P. Nozières, R. Ramazashvili, and A. Tsvelik. The numerical calculations have been carried out using the computing facilities of Theoretical Physics, University of Oxford.

N.A. is grateful the C. Destri and D. Braak for sharing their insights in the course many illuminating discussions, and to A. Ruckenstein for a careful reading of the manuscript.

The research has been supported by the EPSRC grant GR/K97783, the Hungarian Grants OTKA F016604 and OTKA 7283/93, and the U.S-Hungarian Joint Fund No.587.

$\dagger$ Present address: Institut Laue-Langevin.

${ }^{1}$ P. Nozières and A. Blandin, J. Phys. (Paris) 41, 193 (1980).

${ }^{2}$ A. Zawadowski, Phys. Rev. Lett. 45, 211 (1980). K. Vladár and A. Zawadowski, Phys. Rev. B 28, 1564, 1582, 1596 (1983); A. Zawadowski and K. Vladár, in Quantum Tunneling in Condensed Media, edited by Yu. Kagan and A.J. Leggett (North-Holland, Amsterdam, 1992).

${ }^{3}$ D. L. Cox, Phys. Rev. Lett. 59, 1240 (1987).

${ }^{4}$ D. L. Cox and A. Zawadowski, Exotic Kondo Effects in Metals. cond-mat/9704103.

${ }^{5}$ G. Zaránd, Phys. Rev. Lett. 77, 3609 (1996); N. Andrei (unpublished).

${ }^{6}$ N. Andrei and C. Destri, Phys. Rev. Lett. 52, 364 (1984).

${ }^{7}$ C. Destri, Unpublished Notes 1985.

${ }^{8}$ N. Andrei, K. Furuya, and J. H. Lowenstein, Rev. Mod. Phys., 51, 331 (1983).

${ }^{9}$ A. Tsvelik and P. Wiegman, Adv. Phys. 32, 453 (1983).

${ }^{10} \mathrm{~N}$. Andrei, Integrable Models in Condensed Matter Physics, in Series on Modern Condensed Matter Physics - Vol. 6, 458-551, World Scientific, Lecture Notes of ICTP 
Summer Course, September 1992. Editors: S. Lundquist, G. Morandi and $\mathrm{Yu} \mathrm{Lu.}$

${ }^{11}$ A. Tsvelik and P. Wiegman, Z. Phys. B54, 201 (1984).

${ }^{12} \mathrm{P}$. Zinn-Justin and N. Andrei, ENS preprint, condmat/9801158.

${ }^{13}$ S. Okubo, J. Math. Phys. 18, 2382 (1977).

${ }^{14}$ H. F. Jones, Groups, Representations and Physics, The Institute of Physics, 1990.

${ }^{15}$ A. M. Tsvelik, Sov. Phys. JETP 66, 754 (1987).

${ }^{16}$ D. L. Cox and A. E. Ruckenstein, Phys. Rev. Lett. 71, 1613 (1993).

17 J. Gan, N. Andrei, and P. Coleman, Phys. Rev. Lett. 70, 686 (1993).

${ }^{18}$ G. Zaránd, Phys. Rev. B 51, 273 (1995); G. Zaránd and K. Vladár, Phys. Rev. Lett. 76, 2133 (1996).

${ }^{19}$ N. Andrei and A. Jerez, Phys. Rev. Lett. 74, 4507 (1995).

${ }^{20}$ N. Andrei and H. Johannesson, Phys. Lett. 104A, 370 (1984).

${ }^{21}$ V. T. Rajan, Phys. Rev. Lett. 51, 308 (1985).

${ }^{22}$ W. H. Press et al., Numerical Recipes in Fortran, Cambridge University Press, 1992.

${ }^{23}$ H.-U. Desgranges, J. Phys. C, 18, 5481 (1983).

24 J. Kondo, Physica B 84 (Amsterdam), 40 (1976).

${ }^{25}$ A.A. Abrikosov, Physics 2, 5 (1965).

${ }^{26}$ A. Muramatsu and F. Guinea, Phys. Rev. Lett. 57, 2337 (1986).

${ }^{27}$ H. B. Pang and D.L. Cox, Phys. Rev. B 44, 9454 (1991).

${ }^{28}$ A. Moustakas and D. Fisher, Phys. Rev. B. 53, 4300 (1996); ibid. 55, 6832 (1997).

${ }^{29}$ A. Zawadowski et al. (preprint).

${ }^{30}$ For a general introduction see e.g. C. Itzykson and J.B. Zuber, Quantum Field Theory, McGrow-Hill International Editions (Singapore, 1980).

${ }^{31}$ I. Affleck and A. W. W. Ludwig, Nucl. Phys. B 428, 545 (1994).

${ }^{32}$ M. Tornow et al., Physica B 194-196, 1063 (1994).

33 S. Katayama, S. Maekawa, and H. Fukuyama, J. Phys. Soc. Jpn. 56, 697 (1987).

${ }^{34}$ A. Furusaki and K. A. Matveev, Phys. Rev. B 52, 16676 (1995).

35 M. Devoret in Les Houches Summer School on Mesoscopic Quantum Physics, edited by E. Akkermans et al. (NorthHolland, Amsterdam, 1995).

${ }^{36}$ D.V. Averin and K.K. Likharev in Mesoscopic Phenomena in Solids, edited by B.L. Altschuler et al., (North-Holland, 1991). 